\title{
Newton Polytopes and Relative Entropy Optimization
}

\author{
Riley Murray ${ }^{\dagger}$, Venkat Chandrasekaran ${ }^{\dagger, \ddagger}$, and Adam Wierman ${ }^{\dagger} *$ \\ ${ }^{\dagger}$ Department of Computing and Mathematical Sciences \\ $\ddagger$ Department of Electrical Engineering \\ California Institute of Technology \\ Pasadena, CA 91125
}

October 4, 2018

\begin{abstract}
Newton polytopes play a prominent role in the study of sparse polynomial systems, where they help formalize the idea that the root structure underlying sparse polynomials of possibly high degree ought to still be "simple." In this paper we consider sparse polynomial optimization problems, and we seek a deeper understanding of the role played by Newton polytopes in this context. Our investigation proceeds by reparametrizing polynomials as signomials which are linear combinations of exponentials of linear functions in the decision variable - and studying the resulting signomial optimization problems. Signomial programs represent an interesting (and generally intractable) class of problems in their own right. We build on recent efforts that provide tractable relative entropy convex relaxations to obtain bounds on signomial programs. We describe several new structural results regarding these relaxations as well as a range of conditions under which they solve signomial programs exactly. The facial structure of the associated Newton polytopes plays a prominent role in our analysis. Our results have consequences in two directions, thus highlighting the utility of the signomial perspective. In one direction, signomials have no notion of "degree"; therefore, techniques developed for signomial programs depend only on the particular terms that appear in a signomial. When specialized to the context of polynomials, we obtain analysis and computational tools that only depend on the particular monomials that constitute a sparse polynomial. In the other direction, signomials represent a natural generalization of polynomials for which Newton polytopes continue to yield valuable insights. In particular, a number of invariance properties of Newton polytopes in the context of optimization are only revealed by adopting the viewpoint of signomials.
\end{abstract}

Keywords: arithmetic-geometric-mean inequality, certifying nonnegativity, fewnomials, SAGE, signomials, sparse polynomials

*Email: rmurray@caltech.edu,venkatc@caltech.edu, adamw@caltech.edu 


\section{Introduction}

A common representation of an $n$-variate polynomial of degree at-most- $d$ is via a vector of coefficients with respect to the monomial basis

$$
\left\{\boldsymbol{y}^{\boldsymbol{v}}: \boldsymbol{v} \text { in } \mathbb{Z}_{+}^{n}, \mathbf{1}^{\top} \boldsymbol{v} \leq d\right\}
$$

where $\boldsymbol{y}^{\boldsymbol{v}} \doteq \prod_{i=1}^{n} y_{i}^{v_{i}}$. When $d$ is large, one may encounter polynomials of interest with very sparse coefficient vectors. In this situation it becomes more natural to use the representation $\sum_{i=1}^{m} c_{i} \boldsymbol{y}^{\boldsymbol{\alpha}_{i}}$ for exponents $\boldsymbol{\alpha}_{i} \in \mathbb{Z}_{+}^{n}$ and nonzero scalars $c_{i} \in \mathbb{R}$, with $m$ typically being far smaller than $\left(\begin{array}{c}n+d \\ d\end{array}\right)$.

The study of sparse polynomials has a long history. Descartes' Rule of Signs from the 17th century relates the number of positive roots of a univariate polynomial to the number of sign alterations in its vector of coefficients, with the conclusion that a polynomial with $m$ terms has at most $2 m-2$ nonzero real roots (regardless of its degree). In the 20th century, Khovanskii significantly generalized Descartes' result to systems of sparse multivariate polynomials via the theory of fewnomials $[22,23]$. A central geometric object that arises in the course of his analysis is the configuration of the exponent vectors of a polynomial $\sum_{i=1}^{m} c_{i} \boldsymbol{y}^{\boldsymbol{\alpha}_{i}}$ via the associated Newton polytope, which is the set $\mathcal{P}(\boldsymbol{\alpha}) \doteq \operatorname{conv}\left\{\boldsymbol{\alpha}_{i}\right\}_{i=1}^{m}$. The Newton polytope enables an analysis of the root structure of polynomial systems via an appeal to polyhedral geometry, and it helps formalize the idea that the root structure underlying sparse polynomials of possibly high degree ought to be "simple." As an illustration, the celebrated Bernstein-Kushnirenko-Khovanskii (BKK) bound characterizes the number of (complex) roots of a system of polynomials in terms of the mixed volume of the Newton polytopes of the polynomials $[25,6]$.

Where Descartes, Khovanskii, and others studied the root structure of sparse polynomials, we are interested in optimization. Explicit examples of sparse polynomial optimization can be found in system identification [8, 13], model verification [32], chemical and biological processes [14, 18], and electrical engineering $[15,46]$. Polynomial optimization is in general computationally intractable as there are a number of NP-hard problems which can be reduced to minimization of a multivariate polynomial. This motivates a number of natural questions, in the spirit of the previous literature on the root structure of sparse polynomials: Are there methods to obtain bounds on sparse polynomial optimization problems that are computationally tractable and scale efficiently with the number of terms (with no dependence on the degree)? Are there problem families for which these bounds are exact, i.e., solve the problem to global optimality? Which geometric attributes of Newton polytopes play a role in the context of the preceding questions?

We address these questions by considering signomial reparametrizations of polynomials and studying the properties of the resulting signomial optimization problems, where a signomial is a function of the form $\boldsymbol{x} \mapsto \sum_{i=1}^{m} c_{i} \exp \left(\boldsymbol{\alpha}_{i}^{\top} \boldsymbol{x}\right)$ for scalars $c_{i} \in \mathbb{R}$ and exponents $\boldsymbol{\alpha}_{i} \in \mathbb{R}^{n}$. This line of thinking is supported by Khovanskii's original analysis of fewnomials, and it is also present in Bajbar and Stein's recent results on polynomial coercivity [5]. Thus, our paper can also be viewed as a contribution to the literature on signomial optimization, which progressed (and was conceived) independently from developments on the topic of polynomial optimization [42, 33, 27]. In particular, our results highlight the role played by Newton polytopes in the context of signomials (defined again as the convex hull of exponent vectors), which demonstrates their utility in function classes beyond polynomials. Further, we describe families of nonconvex signomial programs which can be globally optimized in an efficient manner. 


\subsection{The Virtues of the Signomial Perspective}

Global minimization is computationally equivalent to the problem of certifying global nonnegativity, and therefore optimizing sparse polynomials can be reduced to the question of checking nonnegativity of sparse polynomials. The point of departure for our discussion is to investigate the related problem of certifying nonnegativity of signomials. One difficulty is that a signomial $\sum_{i=1}^{m} c_{i} \exp \left(\boldsymbol{\alpha}_{i}^{\top} \boldsymbol{x}\right)$ with $\boldsymbol{\alpha}_{i} \in \mathbb{Z}_{+}^{n}$ being globally nonnegative implies only that the corresponding polynomial $\sum_{i=1}^{m} c_{i} \boldsymbol{y}^{\boldsymbol{\alpha}_{i}}$ takes on nonnegative values over the nonnegative orthant, i.e. for $\boldsymbol{y} \in \mathbb{R}_{+}^{n}$. We address this issue by identifying a suitable "signomial representative" of a polynomial, so that nonnegativity of the signomial representative implies global nonnegativity of the polynomial (see Section 5.1). Subject to this point being resolved, the signomial reformulation provides a compelling framework for sparse polynomial optimization. More broadly, signomials with exponent vectors in $\mathbb{R}^{n}$ represent a natural generalization of polynomials for which the lens of Newton polytopes continues to yield valuable insights. We outline here several reasons for the appeal of the signomial perspective.

First, the nonnegativity of a signomial is invariant to any simultaneous nonsingular affine transformation of the exponent vectors. In other words, the precise values of the exponent vectors are less relevant than their positions with respect to each other. This invariance particularly brings to the forefront the combinatorial properties of Newton polytopes such as their face lattice; indeed, this observation manifests itself prominently in our analysis, with our results based primarily on the face structure of Newton polytopes. In contrast, in much of the prior work on Newton polytopes in the context of polynomial optimization, the emphasis in the analysis is mainly driven by the fact that the exponent vectors belong to the integer lattice [36]. Finally, from the viewpoint of sparse polynomial optimization, this invariance property also highlights the fact that the signomial representation of a polynomial is agnostic to the degree of the polynomial and it instead draws attention to the relative locations of the exponent vectors.

Second, there is no countable basis for signomials with exponent vectors in $\mathbb{R}^{n}$, and consequently Newton polytopes and analysis by exponent vectors become critically important for signomials. In particular, the emphasis both in the analysis and in algorithm development for signomial optimization is only on exponent vectors that appear in a signomial. This attribute is ideally suited to investigating sparse polynomial optimization, and to developing computational methods that scale only with the number of terms appearing in a sparse polynomial.

Third, Chandrasekaran and Shah [9] have recently made use of the convex relative entropy function

$$
D(\boldsymbol{\nu}, \boldsymbol{\lambda})=\sum_{i=1}^{m} \nu_{i} \ln \left(\nu_{i} / \lambda_{i}\right) \quad \operatorname{dom}(D)=\mathbb{R}_{+}^{m} \times \mathbb{R}_{+}^{m}
$$

to produce a family of efficiently computable lower bounds that converge to the global optima for broad families of signomial optimization problems. Their work is based on certifying nonnegativity of a signomial $f$ by identifying a decomposition $f=\sum_{i} f_{i}$ with the property that each $f_{i}$ is efficiently certifiable as nonnegative via the arithmetic-geometric inequality. Signomials that possess such a decomposition are termed SAGE functions (sums-of-arithmetic-geometric-exponentials), and checking whether a signomial is SAGE can be formulated as a tractable convex feasibility problem that consists of linear and relative entropy inequalities. This framework provides both a concrete analytical approach for identifying cases in which a nonnegative signomial may be tractably certified as such via convex optimization, and a computational approach for efficiently solving certain families of nonconvex signomial programs. It is also worth mentioning that the landscape of relative entropy solvers (usually known as exponential cone solvers) is rapidly evolving [43, 39, 4]. A recent release 
of MOSEK 9 could solve SAGE relaxations involving millions of exponential cones in mere minutes on a sufficiently powerful workstation.

\subsection{Our Contributions}

Our contributions rest on several key structural results regarding the cone of nonnegative signomials as well as relative entropy certificates of nonnegativity. We now highlight some of these results along with their implications for signomial optimization; these in turn lead to further useful consequences when subsequently specialized to the case of sparse polynomials.

Theorem 2 in Section 3 proves an important sparsity-preserving property of SAGE functions. If a signomial $f$ is SAGE, then there exists a decomposition $f=\sum_{i} f_{i}$ that certifies this property (i.e., each $f_{i}$ is certifiable as nonnegative via the arithmetic-geometric inequality, as described in Section 2), such that each $f_{i}$ consists only of those exponents that appear in $f$. This fundamental attribute of SAGE functions highlights their computational significance - without loss of generality, one need only consider decompositions that involve exponents that are already present in the original signomial. When specialized to the case of polynomials, our results provide an approach for certifying nonnegativity of sparse polynomials in which only the monomials that appear in the polynomial are relevant. Coupled with the computational tractability of checking whether a signomial is SAGE, we obtain a computational framework for sparse polynomial optimization that scales efficiently only with the number of the terms in a polynomial, with no dependence on the degree.

Theorem 4 in Section 3 provides a characterization of the extreme rays of the cone of SAGE functions. In particular, it states that signomials that are efficiently certified as nonnegative by the arithmetic-geometric-inequality - the constituents of a SAGE decomposition - are by themselves not the extreme rays in general; rather, all non-trivial extreme rays of the SAGE cone possess simplicial Newton polytopes. This more refined description of the structure of SAGE functions has a notable consequence when specialized to the case of sparse polynomials. It implies that an appropriate cone of SAGE polynomials (see Section 5 for how these are defined) is equal to the cone of so-called sums of nonnegative circuit polynomials (SONC), which has recently been defined in the literature [20]. However, the cone of SONC polynomials was not known to have any efficient description, and our results provide a computationally tractable formulation for the cone via relative entropy inequalities based on the equivalence to SAGE polynomials; see Theorem 20 in Section 5 for further details.

A third collection of structural results describes when nonnegative signomials are SAGE, with the Newton polytope being the primary subject of these theorems' hypotheses. Theorem 10 is concerned with cases in which the Newton polytope is simplicial, while Theorems 11 and 12 concern when it "decomposes" in an appropriate sense. Each of these theorems exhibits invariance under nonsingular linear transformations of the exponent vectors. The nonnegativity results carry over to signomial optimization in both the unconstrained and constrained cases (Corollaries 14 and 15 respectively), as well as to sparse polynomial optimization (Corollaries 16, 17 and 18). We note that our results depend not only on the facial structure of Newton polytopes but also on the sign pattern of the coefficients (in the spirit of Descartes' Rule of Signs and of Khovanskii's work on fewnomials).

Finally, based on the structure of the underlying Newton polytope, we give a set of conditions under which relative entropy convex relaxations exhibit finite error in signomial optimization problems (Theorem 5). 


\subsection{Related work}

There are three bodies of related work with which we compare and contrast our contributions.

First, the literature on signomial optimization has identified a large number of applications that are fruitfully modeled as signomial programs, but progress on the computational front has been based primarily on either finding local solutions by some form of successive linear/geometric programming approximations [7], or finding global solutions by employing branch-and-bound in conjunction with these previous approximations [41, 40,28]. Our efforts are qualitatively different in that they build on the results in [9] that provide tractable convex relaxations to obtain guaranteed bounds on signomial programs. In particular, the present paper identifies the broadest yet-known families of nonconvex signomial programs that can be efficiently solved via convex relative entropy optimization.

Second, there is a significant body of prior work on convex relaxations for polynomial optimization based on sums-of-squares (SOS) decompositions and semidefinite programming [33, 27, 42]. On the one hand, Hilbert provided a complete characterization of conditions under which nonnegative polynomials are SOS; coupled with the semidefinite description of cones of SOS polynomials, this result identifies classes of polynomial optimization problems that can be solved efficiently via convex optimization. Our results on the exactness of convex relaxations for signomial programming may be viewed as developing a signomial (and sparse polynomial) counterpart, with the role of semidefinite programming (resp. SOS) now being played by relative entropy optimization (resp. SAGE). Further, there have been significant efforts towards developing sparsity-exploiting modifications to the original SOS method have been proposed. ${ }^{1}$ Kojima et. al built on earlier work of Reznick [35] to reduce the size of the SOS matrix variable without loss of generality [24]. Their techniques had meaningful use-cases, but could often fail to perform nontrivial reductions in very modest cases (c.f. Proposition 5.1 of [24]). Subsequently, Waki et. al showed a combinatorial heuristic to induce sparsity in a full-sized SOS matrix variable [45]. Shortly thereafter Nie and Demmel considered a more aggressive approach to replace the order- $\left(\begin{array}{c}n+d \\ d\end{array}\right)$ matrix by a collection of order- $\left(\begin{array}{c}k+d \\ d\end{array}\right)$ matrices for some $k \ll n$ [30]. Very recently, Ahmadi and Majumdar used LP and SOCP approximations of the semidefinite cone to extend SOS to realms where scalability is an issue [2]. Each of the above works suffers from the drawback of exponential runtime dependence on the underlying polynomial degrees. The reason for this poor scaling is that the associated nonnegativity certificates may require monomials that are not present in the original polynomial. In comparison, our results provide a computational framework that does not suffer from this drawback.

Finally, in the context of sparse polynomial optimization, there have been previous efforts aimed at designing alternatives to semidefinite programming for certifying polynomial nonnegativity. Ghasemi et al. $[17,16]$ pioneered the use of geometric programming for this task, but these methods are somewhat limited in scope in terms of the classes of polynomials to which they were applicable. Building on these results, a series of recent papers have proposed certifying nonnegativity of polynomials based on SONC decompositions $[12,19,20,38]$. However, prior to the present paper, there was no known tractable description of the cone of SONC polynomials. In particular, all the existing attempts in the literature for characterizing SONC polynomials are either of exponential size in general or are inexact approximations. By contrast, we give a tractable description based on relative entropy optimization of SAGE polynomials, which are in turn shown to be equivalent to SONC polynomials in Theorem 20 in Section 5.

In summary, our results provide new insights into the power of relative entropy certificates for signomial optimization and signomial nonnegativity. They also provide a compelling sparsitypreserving alternative to semidefinite programming methods for polynomial optimization.

\footnotetext{
${ }^{1}$ The original SOS method uses a matrix variable of order $\left(\begin{array}{c}n+d \\ d\end{array}\right)$; see Section 5.3 for details.
} 


\subsection{Article outline}

Section 2 covers the preliminaries of SAGE functions, which are fundamental to the relative entropy certificates used in this work. Our results begin in Section 3, where we state and prove several new structural results for the cone induced by these nonnegativity certificates. Section 4 concerns exactness results for the relative entropy certificates of signomial nonnegativity, and Section 5 extends these results to the realm of global polynomial nonnegativity. We conclude with Section 6 , which discusses avenues for strengthening the results in this paper.

\subsection{Notation and Conventions}

Vectors and matrices always appear in boldface. The $i^{\text {th }}$ entry of a vector $\boldsymbol{v}$ is $v_{i}$, and the vector formed by deleting the $i^{\text {th }}$ entry from $\boldsymbol{v}$ is $\boldsymbol{v}_{\backslash i}$. We use $\boldsymbol{e}_{i}$ to denote the $i^{\text {th }}$ standard basis vector in $\mathbb{R}^{n}$, and say that matrix $\boldsymbol{A}$ will is built from columns $\boldsymbol{a}_{i}$. The operator $\oplus$ is used for vector concatenation, possibly between vectors of different lengths. Given two vectors $\boldsymbol{a}, \boldsymbol{b}$ the Hadamard product $\boldsymbol{v}=\boldsymbol{a} \odot \boldsymbol{b}$ has entries $v_{i}=a_{i} b_{i}$.

Special sets include the integers from 1 to $\ell$ and the probability simplex in $\mathbb{R}^{n}$ (henceforth denoted $[\ell]$ and $\Delta_{n}$ ). The operators "cl" and "conv" denote computation of a set's closure and convex hull respectively; the operator "ext" returns the extreme points of a compact convex set. In general, subsets of $\mathbb{R}^{n}$ are represented as uppercase letters in Roman font. We use $A+B$ to denote the Minkowski sum of sets $A$ and $B$ within $\mathbb{R}^{n}$. For any convex cone $K$ contained in $\mathbb{R}^{n}$ there is an associated dual cone $K^{\dagger} \doteq\left\{\boldsymbol{y}: \boldsymbol{y}^{\top} \boldsymbol{x} \geq 0\right.$ for all $\boldsymbol{x}$ in $\left.K\right\}$.

Unless otherwise stated, $\boldsymbol{\alpha}$ is a matrix belonging to $\mathbb{R}^{n \times m}$, while $\boldsymbol{c}$ is a vector in $\mathbb{R}^{m}$. Writing $f=\operatorname{Sig}(\boldsymbol{\alpha}, \boldsymbol{c})$ means that $f$ takes values $f(\boldsymbol{x})=\sum_{i=1}^{m} c_{i} \exp \left(\boldsymbol{\alpha}_{i}^{\top} \boldsymbol{x}\right)$. For a fixed signomial $\operatorname{Sig}(\boldsymbol{\alpha}, \boldsymbol{c})$, we often refer to $\boldsymbol{\alpha}_{i} \in \mathbb{R}^{n}$ as an exponent vector, and use $\mathcal{P}(\boldsymbol{\alpha})$ to denote its Newton polytope. The cone of nonnegative signomials over exponents $\boldsymbol{\alpha}$ is

$$
C_{\mathrm{NNS}}(\boldsymbol{\alpha})=\left\{\boldsymbol{c}: \operatorname{Sig}(\boldsymbol{\alpha}, \boldsymbol{c})(\boldsymbol{x}) \geq 0 \text { for all } \boldsymbol{x} \text { in } \mathbb{R}^{n}\right\} .
$$

With the exception of Sections 4.4 and 5.4, all optimization discussed in the present article concerns unconstrained minimization; we use $f^{\star}$ to denote the unconstrained infimum of $f$ over $\mathbb{R}^{n}$.

\section{Background Theory on SAGE Functions}

In their debut, SAGE functions were used as a building block for a hierarchy of convex relaxations to challenging nonconvex signomial optimization problems [9]. Underlying this entire hierarchy were the simple facts that SAGE functions are globally nonnegative, and efficiently recognizable. The purpose of this section is to review the theory of SAGE functions to the extent that it is needed for subsequent development.

Section 2.1 introduces the idea of an AGE function, which serve as the building blocks of SAGE functions. The precise way in which AGE functions extend to SAGE functions is given in Section 2.2. Section 2.3 describes the connection between nonnegativity and optimization in the context of SAGE relaxations. These three sections will be crucial to understanding this article.

\subsection{Simple tests for nonnegativity}

We need some additional structure to make it easier to verify membership in the nonnegativity cone $C_{\mathrm{NNS}}(\boldsymbol{\alpha})$. The structure used by Chandrasekaran and Shah [9] was that the coefficient vector $\boldsymbol{c}$ contained at most one negative entry $c_{k}$; if such a function was globally nonnegative, they called it 
an $A M / G M$ Exponential, or an $A G E$ function, as they employed the arithmetic-geometric inequality to certify its nonnegativity. To facilitate study of such functions, [9] defines the $k^{\text {th }} A G E$ cone

$$
C_{\mathrm{AGE}}(\boldsymbol{\alpha}, k)=\left\{\boldsymbol{c}: \boldsymbol{c}_{\backslash k} \geq \mathbf{0} \text { and } \boldsymbol{c} \text { belongs to } C_{\mathrm{NNS}}(\boldsymbol{\alpha})\right\} .
$$

Some simple properties of $C_{\mathrm{AGE}}(\boldsymbol{\alpha}, k)$ include that (i) it is a closed convex cone, (ii) it contains no lines, and (iii) it contains the nonnegative orthant.

There are two seemingly-distinct but nevertheless-equivalent methods to test membership in $C_{\mathrm{AGE}}(\boldsymbol{\alpha}, k)$. One recognition scheme stems from Reznick's characterization of agiforms [36]. Suppose we are given $\boldsymbol{c}$ with $\boldsymbol{c}_{\backslash k} \geq \mathbf{0}$. If $c_{k} \geq 0$, then $\boldsymbol{c}$ trivially belongs to $C_{\mathrm{AGE}}(\boldsymbol{\alpha}, k)$. If instead $c_{k}<0$, then $\operatorname{Sig}(\boldsymbol{\alpha}, \boldsymbol{c})$ is globally nonnegative iff

$$
\text { some } \quad \boldsymbol{\lambda} \text { in } \Delta_{m} \cap \operatorname{ker} \boldsymbol{e}_{k}^{\top} \text { has } \boldsymbol{\alpha} \boldsymbol{\lambda}=\boldsymbol{\alpha}_{k} \text { and } \prod_{i \neq k}\left[c_{i} / \lambda_{i}\right]^{\lambda_{i}} \geq-c_{k} .
$$

For fixed $\boldsymbol{c}$, verifying the existence of $\boldsymbol{\lambda}$ satisfying (1.1) reduces to solving a geometric program. Although geometric programs are tractable, the lack of joint convexity in $(\boldsymbol{c}, \boldsymbol{\lambda})$ limits the usefulness of such a test. A key contribution of [9] was a test for membership in $C_{\mathrm{AGE}}(\boldsymbol{\alpha}, k)$ that was jointly convex in all relevant variables. Specifically, they showed that a vector $\boldsymbol{c}$ with $\boldsymbol{c}_{\backslash k} \geq \mathbf{0}$ belongs to $C_{\mathrm{AGE}}(\boldsymbol{\alpha}, k)$ if and only if

$$
\text { some } \quad \boldsymbol{\nu}_{\backslash k} \geq \mathbf{0} \text { with } \mathbf{1}^{\top} \boldsymbol{\nu}=0 \text { has } \boldsymbol{\alpha} \boldsymbol{\nu}=\mathbf{0} \text { and } D\left(\boldsymbol{\nu}_{\backslash k}, e \boldsymbol{c}_{\backslash k}\right) \leq c_{k} .
$$

Note that although the necessity of system (1.1) assumes $c_{k}<0$, this assumption is not made in the relative entropy parameterization given by (1.2).

For future reference in the course of our analysis, the dual of the AGE cone is given by

$$
\begin{array}{r}
C_{\mathrm{AGE}}(\boldsymbol{\alpha}, k)^{\dagger}=\operatorname{cl}\left\{\boldsymbol{v}: \boldsymbol{v} \geq 0, \text { and for some } \boldsymbol{\mu}_{k} \text { in } \mathbb{R}^{n}\right. \text { we have } \\
\left.\qquad v_{k} \ln \left(v_{k} / v_{i}\right) \leq\left(\boldsymbol{\alpha}_{k}-\boldsymbol{\alpha}_{i}\right)^{\top} \boldsymbol{\mu}_{k} \text { for } i \text { in }[m]\right\} .
\end{array}
$$

\subsection{From "AGE" to "SAGE"}

We call a signomial a $S A G E$ function if it can be written as a sum of AGE functions. SAGE functions are globally nonnegative by construction - a fact that can be stated in conic form as

$$
\sum_{k \in \mathcal{K}} C_{\mathrm{AGE}}(\boldsymbol{\alpha}, k) \subset C_{\mathrm{NNS}}(\boldsymbol{\alpha})
$$

for any index set $\mathcal{K} \subset[m]$. To best approximate $C_{\text {NNS }}(\boldsymbol{\alpha})$ a first choice is to make $\mathcal{K}$ as large as possible, i.e. $\mathcal{K}=[\mathrm{m}]$. However, it is natural to ask whether one obtain the same approximation of $C_{\mathrm{NNS}}(\boldsymbol{\alpha})$ by taking $\mathcal{K}$ as a proper subset of $[m]$ ?

It turns out that we can, and this observation marks the most basic connection between Newton polytopes and signomial nonnegativity. One can check (see Section 2.4 of [9]) that if $\boldsymbol{\alpha}_{k}$ is extremal in $\mathcal{P}(\boldsymbol{\alpha})$, then any nonnegative signomial $\operatorname{Sig}(\boldsymbol{\alpha}, \boldsymbol{c})$ must have $c_{k} \geq 0$. Taking this as given, we have that if $\boldsymbol{\alpha}_{k}$ is extremal, then the $k^{\text {th }}$ AGE cone with respect to $\boldsymbol{\alpha}$ is simply the nonnegative orthant. Therefore assuming there is at least one nonextremal vector $\boldsymbol{\alpha}_{k}$ in $\mathcal{P}(\boldsymbol{\alpha})$, we can write

$$
C_{\mathrm{SAGE}}(\boldsymbol{\alpha})=\sum_{\boldsymbol{\alpha}_{k} \notin \operatorname{ext} \mathcal{P}(\boldsymbol{\alpha})} C_{\mathrm{AGE}}(\boldsymbol{\alpha}, k) .
$$

We take equation (3) as the definition of $C_{\mathrm{SAGE}}(\boldsymbol{\alpha})$ for the duration of this article. Note that by standard techniques from convex analysis this gives

$$
C_{\mathrm{SAGE}}(\boldsymbol{\alpha})^{\dagger}=\cap_{\boldsymbol{\alpha}_{k} \notin \operatorname{ext} \mathcal{P}(\boldsymbol{\alpha})} C_{\mathrm{AGE}}(\boldsymbol{\alpha}, k)^{\dagger} .
$$


There is a significant conceptual advantage to defining the SAGE cone as in (3). By restricting our attention to $C_{\mathrm{AGE}}(\boldsymbol{\alpha}, k)$ with nonextremal $\boldsymbol{\alpha}_{k}$, we ensure the existence of a solution to $\boldsymbol{\alpha} \boldsymbol{\lambda}=\boldsymbol{\alpha}_{k}$ over $\boldsymbol{\lambda}$ in $\Delta_{m} \cap \operatorname{ker} \boldsymbol{e}_{k}^{\top}$. Thus, under this assumption the $k^{\text {th }}$ AGE cone with respect to $\boldsymbol{\alpha}$ can be defined by either of (1.1) or (1.2). This fact will prove instrumental in characterizing the extreme rays of the SAGE cone in Section 3.

\subsection{From nonnegativity to optimization}

A means for certifying nonnegativity gives rise to a natural method for computing lower bounds for minimization problems: given a function $f$, find the largest constant $\gamma$ where the function $f-\gamma$ can be certified as globally nonnegative. We formalize next this procedure for signomials and SAGE certificates.

Given a signomial $f=\operatorname{Sig}(\boldsymbol{\alpha}, \boldsymbol{c})$ and a constant $\gamma$ in $\mathbb{R}$, we want to check if $f-\gamma$ is SAGE. To do this, we need an unambiguous representation of $f-\gamma$ in terms of $\operatorname{Sig}(\cdot, \cdot)$ notation. Towards this end we assume a-priori that $\boldsymbol{\alpha}$ has $\boldsymbol{\alpha}_{1}=\mathbf{0}$, and we make a point of allowing any entry of $\boldsymbol{c}$ to be zero. Under these assumptions, the function $f-\gamma$ can be written as $\operatorname{Sig}\left(\boldsymbol{\alpha}, \boldsymbol{c}-\gamma \boldsymbol{e}_{1}\right)$. Thus the optimization problem

$$
f_{\mathrm{SAGE}} \doteq \sup \left\{\gamma: \boldsymbol{c}-\gamma \boldsymbol{e}_{1} \text { in } C_{\mathrm{SAGE}}(\boldsymbol{\alpha})\right\}
$$

is well defined, and its optimal value satisfies $f_{\text {SAGE }} \leq f^{\star}$. We also analyze the dual problem to (5), and for reference, we obtain via conic duality that

$$
f_{\mathrm{SAGE}}=\inf \left\{\boldsymbol{c}^{\top} \boldsymbol{v}: \boldsymbol{e}_{1}^{\top} \boldsymbol{v}=1, \boldsymbol{v} \text { in } C_{\mathrm{SAGE}}(\boldsymbol{\alpha})^{\dagger}\right\} .
$$

Although not done in [9], it can be shown that strong duality holds in the primal-dual pair (5)-(6). This fact is important for our later theorems, and so we make a point to state it clearly in the following proposition:

Proposition 1. Strong duality always holds in the computation of $f_{\mathrm{SAGE}}$.

The proposition is proven in Appendix 7.2 using convex analysis.

\section{Structural Results for SAGE Certificates}

This section presents a variety of new geometric results and analytical characterizations on the SAGE cone and on SAGE relaxations for signomial minimization. The first two of these results will have applications to polynomial nonnegativity, as discussed later in Section 5. Statements of the theorems are provided below, along with discussion of the theorems' significance. Proofs are deferred to later subsections.

\subsection{Main results}

Our first theorem shows that when checking if $\boldsymbol{c}$ belongs to $C_{\mathrm{SAGE}}(\boldsymbol{\alpha})$ we can restrict the search space of SAGE decompositions to those exhibiting a very particular structure. It highlights the sparsity-preserving property of SAGE, and in so doing has significant implications for both the practicality of solving SAGE relaxations, and Section 5's development of SAGE polynomials.

Theorem 2. If $\boldsymbol{c}$ is a vector in $C_{\mathrm{SAGE}}(\boldsymbol{\alpha})$ with nonempty set $\mathcal{N} \doteq\left\{i: c_{i}<0\right\}$, then exist vectors $\left\{\boldsymbol{c}^{(i)}\right\}_{i \in \mathcal{N}}$ satisfying

$$
\text { 1. } c=\sum_{i \in \mathcal{N}} c^{(i)},
$$


2. $\boldsymbol{c}^{(i)} \in C_{\mathrm{AGE}}(\boldsymbol{\alpha}, i)$, and

3. $c_{j}^{(i)}=0$ for all $i \neq j$ in $\mathcal{N}$.

We can use Theorem 2 to define some parameterized AGE cones that will be of use to us in Section 4. Specifically, for an index set $I$ contained within $[m]$, and an index $k$ in $[m]$, define

$$
C_{\mathrm{AGE}}(\boldsymbol{\alpha}, k, I)=\left\{\boldsymbol{c}: \boldsymbol{c} \text { in } C_{\mathrm{AGE}}(\boldsymbol{\alpha}, k), c_{i}=0 \text { for all } i \text { in } I \backslash\{k\}\right\} .
$$

In terms of such sets we have the following corollary of Theorem 2 .

Corollary 3. A signomial $f=\operatorname{Sig}(\boldsymbol{\alpha}, \boldsymbol{c})$ with $\boldsymbol{\alpha}_{1}=\mathbf{0}$ has

$$
f_{\mathrm{SAGE}}=\sup \left\{\gamma: \boldsymbol{c}-\gamma \boldsymbol{e}_{1} \text { in } \sum_{k \in \mathcal{N} \cup\{1\}} C_{\mathrm{AGE}}(\boldsymbol{\alpha}, k, \mathcal{N})\right\}
$$

for both $\mathcal{N}=\left\{i: c_{i}<0\right\}$ and $\mathcal{N}=\left\{i: c_{i} \leq 0\right\}$.

This corollary has two implications for practical algorithms for signomial optimization. First, it shows that for $k=\left|\left\{i: c_{i}<0\right\}\right|$, computing $f_{\text {SAGE }}$ can easily be accomplished with a relative entropy program of size $O(\mathrm{~km})$; this is a dramatic improvement over the naïve implementation for computing $f_{\mathrm{SAGE}}$, which would involve a relative entropy program of size $O\left(\mathrm{~m}^{2}\right)$. Second the improved conditioning resulting from restricting the search space in this way often makes the difference in whether existing solvers can handle SAGE relaxations of moderate size. This point is brought to the forefront in recent experimental demonstrations of relative entropy relaxations; the authors of [21] discuss various preprocessing strategies to enable faster solution of such optimization problems.

Our next theorem characterizes the extreme rays of the SAGE cone. To describe these extreme rays, we use a notion from matroid theory [31]: a set of points $X=\left\{\boldsymbol{x}_{i}\right\}_{i=1}^{\ell}$ is called a circuit if it is affinely dependent, but any proper subset $\left\{\boldsymbol{x}_{i}\right\}_{i \neq k}$ is affinely independent. If a circuit with $\ell$ elements contains $\ell-1$ extreme points, then we say that the circuit is simplicial.

Theorem 4. Every extreme ray of a $S A G E$ cone is supported on either a single coordinate, or a set of coordinates inducing a simplicial circuit.

Theorem 4 admits a partial converse: any set of coordinates $\mathcal{I}$ inducing a simplicial circuit $\left\{\boldsymbol{\alpha}_{i}\right\}_{i \in \mathcal{I}}$ supports a family of extreme rays in the SAGE cone. Note that the number of such index sets can be exponential in $n$, depending on the structure of $\boldsymbol{\alpha}$. By comparison, the standard description of $C_{\mathrm{SAGE}}(\boldsymbol{\alpha})$ involves a relative entropy program only of size $O\left(m^{2}\right)$. Hence Theorem 4 shows that the standard relative entropy extended formulation of the SAGE cone is extremely efficient. When specialized to the context of polynomials, this result gives us an equivalence between SAGE polynomials (suitably defined in Section 5) and the previously defined SONC polynomials [19], thus providing an efficient description of the latter set which was not known to be tractable.

Where the previous two theorems addressed SAGE as a means of certifying nonnegativity, this section's final theorem directly considers SAGE as a relaxation scheme for signomial minimization. Specifically, it exploits the primal formulation for $f_{\text {SAGE }}$ to establish sufficient conditions under which SAGE relaxations can only exhibit finite error.

Theorem 5. Suppose there exists an $\epsilon>0$ so that $(1+\epsilon) \boldsymbol{\alpha}_{j}$ belongs to $\mathcal{P}(\boldsymbol{\alpha})$ for all nonextremal $\boldsymbol{\alpha}_{j}$. Then $f=\operatorname{Sig}(\boldsymbol{\alpha}, \boldsymbol{c})$ is bounded below iff $f_{\mathrm{SAGE}}$ is finite.

The hypothesis of Theorem 5 is significantly weaker than those found elsewhere in this work, and it is especially notable as we do not know of analogous theorems in the literature on SOS relaxations for polynomial optimization. 


\subsection{Proof of the restriction theorem for SAGE decompositions (Theorem 2)}

We begin our proof with two lemmas that allow us to remove many potential decompositions of SAGE functions from consideration.

Lemma 6. A vector $\boldsymbol{c}$ with at least one negative entry belongs to $C_{\mathrm{SAGE}}(\boldsymbol{\alpha})$ iff

$$
\boldsymbol{c} \in \sum_{i: c_{i}<0} C_{\mathrm{AGE}}(\boldsymbol{\alpha}, i) .
$$

Proof. Fix $\boldsymbol{c}$ in $C_{\mathrm{SAGE}}(\boldsymbol{\alpha})$ with SAGE decomposition $\boldsymbol{c}=\sum_{i \in \mathcal{D}} \boldsymbol{c}^{(i)}$ for some index set $\mathcal{D}$. If $\mathcal{D}=\left\{i: c_{i}<0\right\}$, then there is nothing to prove, so suppose there is some $k$ in $\mathcal{D}$ with $c_{k} \geq 0$. We construct an alternative decomposition of $\boldsymbol{c}$ using only cones $C_{\mathrm{AGE}}(\boldsymbol{\alpha}, i)$ with $i$ in $\mathcal{D} \backslash\{k\}$.

The construction depends on the sign of $c_{k}^{(k)}$. If $c_{k}^{(k)}$ is nonnegative then the problem of removing dependence on $C_{\mathrm{AGE}}(\boldsymbol{\alpha}, k)$ is trivial: for $i$ in $\mathcal{D} \backslash\{k\}$, the vectors

$$
\tilde{\boldsymbol{c}}^{(i)}=\boldsymbol{c}^{(i)}+\boldsymbol{c}^{(k)} /(|\mathcal{D}|-1)
$$

belong to $C_{\mathrm{AGE}}(\boldsymbol{\alpha}, i)$, and sum to $\boldsymbol{c}$. If instead $c_{k}^{(k)}<0$, then there exists some index $i \neq k$ in $\mathcal{D}$ with $c_{k}^{(i)}$ positive. This allows us to define the distribution $\boldsymbol{\lambda}$ with $\lambda_{i}=c_{k}^{(i)} / \sum_{j \in \mathcal{D} \backslash\{k\}} c_{k}^{(j)}$ for $i \neq k$ in $\mathcal{D}$. With $\boldsymbol{\lambda}$ we construct the $|\mathcal{D}|-1$ vectors

$$
\tilde{\boldsymbol{c}}^{(i)}=\boldsymbol{c}^{(i)}+\lambda_{i} \boldsymbol{c}^{(k)} .
$$

We claim that for every $i \neq k$ in $\mathcal{D}$, the coordinate $\tilde{c}_{k}^{(i)}$ is nonnegative. This is certainly true when $\lambda_{i}=0$, but more importantly, $\lambda_{i}>0$ implies

$$
\frac{1}{\lambda_{i}} \tilde{c}_{k}^{(i)}=\frac{1}{\lambda_{i}}\left(c_{k}^{(i)}+\lambda_{i} c_{k}^{(k)}\right)=\left[\sum_{j \in \mathcal{D} \backslash\{k\}} c_{k}^{(j)}\right]+c_{k}^{(k)}=c_{k} \geq 0 .
$$

Hence $\boldsymbol{c}$ can be expressed as the sum of vectors $\left\{\tilde{\boldsymbol{c}}^{(i)}\right\}_{i \in \mathcal{D} \backslash\{k\}}$ where each vector $\tilde{\boldsymbol{c}}^{(i)}$ belongs to $C_{\mathrm{AGE}}(\boldsymbol{\alpha}, i)$.

From here, update $\mathcal{D} \leftarrow \mathcal{D} \backslash\{k\}$. If $\mathcal{D}$ contains another index $k^{\prime}$ with $c_{k^{\prime}} \geq 0$, then repeat the above procedure to remove the unnecessary AGE cone. Naturally, this process continues until $\mathcal{D}=\left\{i: c_{i}<0\right\}$.

Lemma 7. Let $\boldsymbol{a}, \boldsymbol{b}$ be vectors in $\mathbb{R}^{n}$ with distinguished indices $i \neq j$ so that

$$
\boldsymbol{a}_{\backslash i}, \boldsymbol{b}_{\backslash j} \geq \mathbf{0} \quad \text { and } \quad a_{k}+b_{k}<0 \text { for } k \text { in }\{i, j\} .
$$

Then by a conic combination of $\{\boldsymbol{a}, \boldsymbol{b}\}$ we can obtain vectors $\hat{\boldsymbol{a}}, \hat{\boldsymbol{b}}$ satisfying

$$
\hat{\boldsymbol{a}}+\hat{\boldsymbol{b}}=\boldsymbol{a}+\boldsymbol{b} \quad \text { and } \quad \hat{a}_{j}=\hat{b}_{i}=0 .
$$

Proof. By reindexing, take $i=1$ and $j=2$. Such $\hat{\boldsymbol{a}}, \hat{\boldsymbol{b}}$ exist if and only if some $\boldsymbol{\lambda}$ in $\mathbb{R}_{+}^{4}$ solves

$$
\left[\begin{array}{cccc}
a_{1} & 0 & b_{2} & 0 \\
0 & a_{2} & 0 & b_{1} \\
1 & 1 & 0 & 0 \\
0 & 0 & 1 & 1
\end{array}\right]\left[\begin{array}{c}
\lambda_{1} \\
\lambda_{2} \\
\lambda_{3} \\
\lambda_{4}
\end{array}\right]=\left[\begin{array}{l}
0 \\
0 \\
1 \\
1
\end{array}\right] .
$$


The determinant of the matrix above is $d=a_{1} b_{2}-b_{1} a_{2}$. If $a_{2}$ or $b_{1}=0$, then $d>0$. If $a_{2}, b_{1} \neq 0$, then $d>0 \Leftrightarrow\left|b_{2} / a_{2}\right| \cdot\left|a_{1} / b_{1}\right|>1$. In this case we use the assumptions on $\boldsymbol{a}, \boldsymbol{b}$ to establish the slightly stronger condition that $\left|b_{2} / a_{2}\right|>1$ and $\left|a_{1} / b_{1}\right|>1$. In both cases we have a nonzero determinant, so there exists a unique $\boldsymbol{\lambda}$ in $\mathbb{R}^{4}$ satisfying system (7). Now we need only prove that this $\boldsymbol{\lambda}$ is nonnegative.

One may verify that the symbolic solution to $(7)$ is

$$
\begin{aligned}
& \lambda_{1}=-\left(a_{2}+b_{2}\right) b_{1} / d, \quad \lambda_{2}=\left(a_{1}+b_{1}\right) b_{2} / d \\
& \lambda_{3}=a_{1}\left(a_{2}+b_{2}\right) / d, \quad \lambda_{4}=-\left(a_{1}+b_{1}\right) a_{2} / d
\end{aligned}
$$

and furthermore that all numerators and denominators are nonnegative.

With Lemmas 6 and 7 in hand, the proof of Theorem 2 essentially reduces to Gaussian elimination with back-substitution.

Theorem 2. Let $\boldsymbol{c}$ be a vector in $C_{\mathrm{SAGE}}(\boldsymbol{\alpha})$ with $k$ negative entries $c_{1}, \ldots, c_{k}$.

By Lemma 6 , there exists an $k$-by-m matrix $\boldsymbol{C}$ with $i^{\text {th }}$ row in $C_{\mathrm{AGE}}(\boldsymbol{\alpha}, i)$, and $\boldsymbol{c}=\boldsymbol{C}^{\boldsymbol{\top}} \mathbf{1}$. We repeatedly apply Lemma 7 as row operations to reduce $\boldsymbol{C}$ to the form $[\boldsymbol{U} \mid \boldsymbol{M}]$ with upper-triangular $\boldsymbol{U}$ and dense $\boldsymbol{M}$. After this is done, we once again apply Lemma 7 as row operations to eliminate the strictly upper triangular part of $\boldsymbol{U}$. Because these operations preserve column sums, we have

$$
\boldsymbol{C} \cong \tilde{\boldsymbol{C}}=\left[\operatorname{diag}\left(c_{1}, \ldots, c_{k}\right) \mid \boldsymbol{W}\right]
$$

with dense $\boldsymbol{W}$. Moreover, since the row operations only involve conic combinations of AGE vectors, every row of $\tilde{\boldsymbol{C}}$ defines a nonnegative signomial, and the matrix $\boldsymbol{W}$ is nonnegative. It follows that the $i^{\text {th }}$ row of $\tilde{\boldsymbol{C}}$ belongs to $C_{\mathrm{AGE}}(\boldsymbol{\alpha}, i)$, and furthermore that $\boldsymbol{c}=\tilde{\boldsymbol{C}}^{\top} \mathbf{1}$.

\subsection{Proof of extreme ray characterization of the SAGE cone (Theorem 4)}

Because every ray in the SAGE cone (extreme or otherwise) can be written as a sum of rays in AGE cones, it suffices to characterize the extreme rays of AGE cones. For the duration of this section we discuss the AGE cone $C_{\mathrm{AGE}}(\boldsymbol{\alpha}, k)$, where $\boldsymbol{\alpha}_{k}$ is nonextremal in $\mathcal{P}(\boldsymbol{\alpha})$.

It can easily be shown that for any index $i$ in $[m]$, the ray $\left\{r \boldsymbol{e}_{i}: r \geq 0\right\}$ is extremal in $C_{\mathrm{AGE}}(\boldsymbol{\alpha}, k)$. We call these these rays (those supported on a single coordinate) the trivial extreme rays of the AGE cone. The bulk of the proof of Theorem 4 is to establish that all nontrivial extreme rays of the AGE cone are supported on simplicial circuits. We begin with our first lemma regarding representations of vectors in the intersection of an affine subspace and the probability simplex.

Lemma 8. Fix $\boldsymbol{Y}$ in $\mathbb{R}^{n \times m}, \boldsymbol{x}$ in $\mathbb{R}^{n}$, and define $\Lambda_{x}=\Delta_{m} \cap\{\boldsymbol{\lambda}: \boldsymbol{x}=\boldsymbol{Y} \boldsymbol{\lambda}\}$. If $\boldsymbol{\lambda}$ in $\Lambda_{x}$ has nonsimplicial $\left\{\boldsymbol{y}_{i}\right\}_{i \in \operatorname{supp}(\boldsymbol{\lambda})}$, then there exists a scalar $z$ in $(0,1)$ and vectors $\boldsymbol{\lambda}^{(1)}, \boldsymbol{\lambda}^{(2)}$ in $\Lambda_{x}$ with $\boldsymbol{\lambda}=z \boldsymbol{\lambda}^{(1)}+(1-z) \boldsymbol{\lambda}^{(2)}$ and $\operatorname{supp} \boldsymbol{\lambda}^{(i)} \subsetneq \operatorname{supp} \boldsymbol{\lambda}$.

When we invoke Lemma 8 to establish Theorem 4 , it is applied in a recursive manner to construct particular vectors $\left\{\boldsymbol{\lambda}^{(i)}\right\}_{i}$ and a distribution $\boldsymbol{z}$ so that $\boldsymbol{\lambda}=\sum_{i} z_{i} \boldsymbol{\lambda}^{(i)}$. We choose to frame the lemma is this generic way in order to separate proofs involving classical convex analysis from proofs involving the SAGE cone.

Proof. Let $\boldsymbol{\lambda}$ belonging to $\Lambda_{x}$ have nonsimplicial $\left\{\boldsymbol{y}_{i}: i\right.$ in $\left.\operatorname{supp} \boldsymbol{\lambda}\right\}$. By Minkowski-Caratheodory, there exists at least one $\boldsymbol{\lambda}^{(1)}$ in $\Lambda_{x}$ with supp $\boldsymbol{\lambda}^{(1)} \subset \operatorname{supp} \boldsymbol{\lambda}$ and simplicial $\left\{\boldsymbol{y}_{i}: i\right.$ in $\left.\operatorname{supp} \boldsymbol{\lambda}^{(1)}\right\}$. We will use $\boldsymbol{\lambda}$ and $\boldsymbol{\lambda}^{(1)}$ to construct the desired $\boldsymbol{\lambda}^{(2)}$ and $z$. 
For each real $t$, consider $\boldsymbol{\lambda}_{t}^{\prime} \doteq \boldsymbol{\lambda}^{(1)}+t\left(\boldsymbol{\lambda}-\boldsymbol{\lambda}^{(1)}\right)$. It is easy to see that for all $t$ the vector $\boldsymbol{\lambda}_{t}^{\prime}$ belongs to the affine subspace $\left\{\boldsymbol{w}: \boldsymbol{x}=\boldsymbol{Y} \boldsymbol{w}, \mathbf{1}^{\top} \boldsymbol{w}=1\right\}$, and furthermore the support of $\boldsymbol{\lambda}_{t}^{\prime}$ is contained within the support of $\boldsymbol{\lambda}$. Now define $T=\max \left\{t: \lambda_{t}^{\prime}\right.$ in $\left.\Delta_{m}\right\}$; we claim that $T>1$ and that the support of $\boldsymbol{\lambda}_{T}^{\prime}$ is a proper subset of the support of $\boldsymbol{\lambda}$. The latter claim is more or less immediate. To establish the former claim consider how $\boldsymbol{\lambda}_{t}^{\prime}$ (as an affine combination of $\boldsymbol{\lambda}^{(1)}, \boldsymbol{\lambda}$ ) belongs to $\Delta_{m}$ if and only if it is elementwise nonnegative. This lets us write $T=\max \left\{t: \boldsymbol{\lambda}_{t}^{\prime} \geq \mathbf{0}\right\}$. Next, use our knowledge about the support of $\boldsymbol{\lambda}_{t}^{\prime}$ to rewrite the constraint " $\boldsymbol{\lambda}_{t}^{\prime} \geq \mathbf{0}$ " as " $\lambda_{i}^{(1)}+t\left(\lambda_{i}-\lambda_{i}^{(1)}\right) \geq$ 0 for all $i$ in $\operatorname{supp} \boldsymbol{\lambda}$." Once written in this form, we see that for $t=1$ all constraints are satisfied strictly. It follows that $T>1$ at optimality, and furthermore that the support of $\boldsymbol{\lambda}_{T}^{\prime}$ is distinct from (read: a proper subset of) that of $\boldsymbol{\lambda}$.

We complete the proof by setting $\boldsymbol{\lambda}^{(2)}=\boldsymbol{\lambda}_{T}^{\prime}$ and $z=1-1 / T$.

Now our analysis returns explicitly to AGE cones; let $c$ be a vector defining a nontrivial extreme ray $R=\{r \boldsymbol{c}: r \geq 0\}$ in $C_{\mathrm{AGE}}(\boldsymbol{\alpha}, k)$. By assumption that $R$ is nontrivial we have $c_{k}<0$, and hence the existence of an AM/GM witness $\boldsymbol{\lambda}$ satisfying (1.1). The next lemma says that if $\boldsymbol{\lambda}$ can be represented as a mixture of simpler convex combinations $\left\{\boldsymbol{\lambda}^{(i)}\right\}_{i=1}^{\ell}$ satisfying $\boldsymbol{\alpha} \boldsymbol{\lambda}^{(i)}=\boldsymbol{\alpha}_{k}$, then $\boldsymbol{c}^{\prime}$ 's membership in $C_{\mathrm{AGE}}(\boldsymbol{\alpha}, k)$ can be certified by the AM/GM witnesses $\boldsymbol{\lambda}^{(i)}$.

Lemma 9. Let $\boldsymbol{\lambda}$ denote an $A M / G M$ witness for $\boldsymbol{c} \in C_{\mathrm{AGE}}(\boldsymbol{\alpha}, k)$. Then for any $\boldsymbol{z}$ in $\Delta_{\ell}$ and $\left\{\boldsymbol{\lambda}^{(i)}\right\}_{i=1}^{\ell} \subset \Delta_{m} \cap$ ker $\boldsymbol{e}_{k}^{\top}$ satisfying

$$
\boldsymbol{\lambda}=\sum_{i=1}^{\ell} z_{i} \boldsymbol{\lambda}^{(i)} \quad \text { and } \quad \boldsymbol{\alpha} \boldsymbol{\lambda}^{(i)}=\boldsymbol{\alpha}_{k} \text { for each } i \text { in }[\ell],
$$

there exist vectors $\left\{\boldsymbol{c}^{(i)}\right\}_{i=1}^{\ell} \subset C_{\mathrm{AGE}}(\boldsymbol{\alpha}, k)$ satisfying

$$
\boldsymbol{c} \geq \sum_{i=1}^{\ell} z_{i} \boldsymbol{c}^{(i)} \quad \text { and } \quad \operatorname{supp} \boldsymbol{c}^{(i)}=\{k\} \cup \operatorname{supp} \boldsymbol{\lambda}^{(i)} \text { for each } i \text { in }[\ell] .
$$

Proof. Given an AM/GM witness $\boldsymbol{\lambda}$ for $\boldsymbol{c} \in C_{\mathrm{AGE}}(\boldsymbol{\alpha}, k)$, as well as the associated vectors $\left\{\boldsymbol{\lambda}^{(i)}\right\}_{i=1}^{\ell}$ and $\boldsymbol{z}$, define

$$
\begin{aligned}
c_{j}^{(i)} & =\left\{\begin{array}{ll}
c_{j} \lambda_{j}^{(i)} / \lambda_{j} & \text { if } \lambda_{j}>0 \\
0 & \text { if otherwise }
\end{array} \text { for } j \neq k,\right. \text { and } \\
c_{k}^{(i)} & =-\prod_{j \neq k}\left[c_{j}^{(i)} / \lambda_{j}^{(i)}\right]^{\lambda_{j}^{(i)}} .
\end{aligned}
$$

Note that $\boldsymbol{z}$ does not play an explicit role in the definition of the $\boldsymbol{c}^{(i)}$. Nevertheless, one may verify that for each $i$ in $[\ell]$, the pairs $\left(\boldsymbol{c}^{(i)}, \boldsymbol{\lambda}^{(i)}\right)$ satisfy (1.1) by construction, and so each $\boldsymbol{c}^{(i)}$ belongs to $C_{\mathrm{AGE}}(\boldsymbol{\alpha}, k)$. The lemma's claim regarding the supports of the $\boldsymbol{c}^{(i)}$ (relative to those of the $\left.\boldsymbol{\lambda}^{(i)}\right)$ is likewise apparent.

Now we need to show $\sum_{i=1}^{\ell} z_{i} c_{j}^{(i)} \leq c_{j}$ for all indices $j$ in $[m]$. Direct substitution shows that this inequality actually holds with equality at all coordinate indices $j \neq k$, so we can focus on proving the inequality at $j=k$. Beginning with the definitions of $\boldsymbol{\lambda}, \boldsymbol{\lambda}^{(i)}$, and $\boldsymbol{z}$, we can write

$$
-c_{k} \leq \prod_{j \neq k}\left[c_{j} / \lambda_{j}\right]^{\lambda_{j}}=\prod_{j \neq k} \prod_{i=1}^{\ell}\left[c_{j} / \lambda_{j}\right]^{z_{i} \lambda_{j}^{(i)}}=\prod_{i=1}^{\ell}\left(\prod_{j \neq k}\left[c_{j} / \lambda_{j}\right]^{\lambda_{j}^{(i)}}\right)^{z_{i}}
$$


where the last equality interchanges the order of the products, and redraws parenthesis. Next, use the defining properties of $\left(\boldsymbol{c}^{(i)}, \boldsymbol{\lambda}^{(i)}\right)$ to obtain

$$
\prod_{j \neq k}\left[c_{j} / \lambda_{j}\right]^{\lambda_{j}^{(i)}}=\prod_{j \neq k}\left[c_{j}^{(i)} / \lambda_{j}^{(i)}\right]^{\lambda_{j}^{(i)}}=-c_{k}^{(i)} \geq 0 \quad \text { for every } i \text { in }[\ell] .
$$

Combine (9) with (8) via the AM/GM inequality to produce

$$
-c_{k} \leq \prod_{i=1}^{\ell}\left[-c_{k}^{(i)}\right]^{z_{i}} \leq \sum_{i=1}^{\ell} z_{i}\left[-c_{k}^{(i)}\right]
$$

as desired.

With Lemmas 8 and 9 in hand, we can prove Theorem 4. At a high level, the idea is to recursively apply Lemma 8 until we arrive at a collection of AM/GM witnesses that are supported only on simplicial circuits. From there, we apply Lemma 9 to demonstrate that $\boldsymbol{c}$ can be decomposed as a sum of trivial and simplicial-circuit AGE vectors.

Theorem 4. Let $k$ be the index of some nonextremal $\boldsymbol{\alpha}_{k}$ in $\mathcal{P}(\boldsymbol{\alpha})$, and fix $\boldsymbol{c}$ in $C_{\mathrm{AGE}}(\boldsymbol{\alpha}, k)$ with AM/GM witness $\boldsymbol{\lambda}$ in $\Delta_{m} \cap$ ker $\boldsymbol{e}_{k}^{\top}$. Define $\boldsymbol{Y}$ as the matrix formed by stacking $\boldsymbol{\alpha}$ on top of $\boldsymbol{e}_{k}$ (the $k^{\text {th }}$ standard-basis vector in $\mathbb{R}^{m}$ ), and define $\boldsymbol{x}$ as the vector in $\mathbb{R}^{n+1}$ formed by appending a zero to $\boldsymbol{\alpha}_{k}$. Now apply Lemma 8 repeatedly with this choice of $\boldsymbol{Y}$ and $\boldsymbol{x}$ to recover $\left\{\boldsymbol{\lambda}^{(i)}\right\}_{i=1}^{\ell} \subset \Delta_{m} \cap \operatorname{ker} \boldsymbol{e}_{k}^{\top}$ and $\boldsymbol{z}$ in $\Delta_{\ell}$ where

- $\boldsymbol{Y} \boldsymbol{\lambda}^{(i)}=\boldsymbol{x}$ for $i$ in $[\ell]$,

- $A_{i} \doteq\left\{\boldsymbol{y}_{j}: \lambda_{j}^{(i)}>0\right\}$ is simplicial for every $i$ in $[\ell]$, and

- $\boldsymbol{\lambda}=\sum_{i=1}^{\ell} z_{i} \boldsymbol{\lambda}^{(i)}$.

A key property of these $\boldsymbol{\lambda}^{(i)}$ 's is that since $A_{i}$ is simplicial, it must be that $\boldsymbol{x}$ lays in the relative interior of conv $A_{i}$. By exploiting this fact, we see that the points $\left\{\boldsymbol{\alpha}_{k}\right\} \cup A_{i}$ comprise a simplicial circuit.

We use the vectors $\boldsymbol{\lambda}^{(i)}$ to build a decomposition of $\boldsymbol{c}$. Invoking Lemma 9 on $\left\{\boldsymbol{\lambda}^{(i)}\right\}_{i=1}^{\ell}$ and $\boldsymbol{z}$, there exist $\boldsymbol{c}^{(i)}$ 's in $C_{\mathrm{AGE}}(\boldsymbol{\alpha}, k)$ with supp $\boldsymbol{c}^{(i)}=\{k\} \cup \operatorname{supp} \boldsymbol{\lambda}^{(i)}$ and $\sum_{i=1}^{\ell} z_{i} \boldsymbol{c}^{(i)} \leq \boldsymbol{c}$. The former property ensures that $\boldsymbol{c}^{(i)}$ are supported on simplicial circuits, while the latter property implies the existence of some $\boldsymbol{c}^{(+)} \geq \mathbf{0}$ satisfying

$$
\boldsymbol{c}=\boldsymbol{c}^{(+)}+\sum_{i=1}^{\ell} \boldsymbol{c}^{(i)} .
$$

That is, $\boldsymbol{c}$ can be decomposed into a sum vectors along the AGE cone's trivial extreme rays, and AGE vectors supported on simplicial circuits.

We have accomplished the key step in the proof: nontrivial extreme rays of $C_{\mathrm{AGE}}(\boldsymbol{\alpha}, k)$ can be supported on index sets no larger than those inducing simplicial circuits. To finish the proof we need to establish that no nontrivial extreme ray can be supported on an index set inducing a proper subset of a simplicial circuit. This step follows almost immediately from the definition that a circuit is a minimal affinely-dependent set. As such, if $c$ has support in a proper subset of a simplicial circuit, we would have that its support is over indices $j$ where every $\boldsymbol{\alpha}_{j}$ is extremal. If this were the case then we could not have $c_{k}<0$, and so $c$ could not define a nontrivial extreme ray.

Theorem 4 is of great use to us in Section 5 when we introduce and analyze the idea of a $S A G E$ polynomial. 


\subsection{Proof of finite error in SAGE relaxations (Theorem 5)}

Understanding the gap $f_{\mathrm{SAGE}}-f^{\star}$ is in general very challenging, but the primal-form relative entropy program for computing $f_{\mathrm{SAGE}}$ provides some unique traction at least for characterizing finiteness of this error.

Theorem 5. Let $f=\operatorname{Sig}(\boldsymbol{\alpha}, \boldsymbol{c})$ have $\boldsymbol{\alpha}_{1}=\mathbf{0}$ and $f^{\star}>-\infty$. We may assume without loss of generality that $c_{1}=0$. Use $\mathcal{E}=\left\{i: \boldsymbol{\alpha}_{i}\right.$ nonzero, extremal $\}$ to denote extremal exponents of $f$, excluding the possibly-extremal exponent $\boldsymbol{\alpha}_{1}=\mathbf{0}$. The desired claim holds if there exists a positive constant $\gamma$ so that the translate $f_{\gamma}=f+\gamma$ is SAGE.

Define $\hat{\boldsymbol{c}}=\boldsymbol{c}+\gamma \boldsymbol{e}_{1}$ as the coefficient vector of $f_{\gamma}$. Because $f^{\star}>-\infty$ we have $c_{i}=\hat{c}_{i} \geq 0$ for every $i$ in $\mathcal{E}$. Let $\mathcal{N}$ denote the set of indices $i$ for which $\hat{c}_{i}<0$. For each such index $i$ we define the vector $\hat{\boldsymbol{c}}^{(i)}$ in $\mathbb{R}^{m}$ by

$$
\hat{c}_{j}^{(i)}= \begin{cases}\hat{c}_{i} & \text { if } i=j \\ \hat{c}_{j} /|\mathcal{N}| & \text { if } i \neq j \text { and } j \text { not in } \mathcal{N} . \\ 0 & \text { if } i \neq j \text { and } j \text { in } \mathcal{N}\end{cases}
$$

Certainly, $\sum_{i \in \mathcal{N}} \hat{\boldsymbol{c}}^{(i)}=\hat{\boldsymbol{c}}$ and $\hat{\boldsymbol{c}}_{\backslash i}^{(i)}$ is nonnegative (in particular $\hat{c}_{1}^{(i)}=\gamma /|\mathcal{N}|$ is positive).

Now we build the vectors $\boldsymbol{\nu}^{(i)}$ for $i$ in $\mathcal{N}$. Because $\mathcal{N}$ is contained within $[m] \backslash \mathcal{E}$, we have that each $i$ in $\mathcal{N}$ satisfies $(1+\epsilon) \boldsymbol{\alpha}_{i}$ in $\mathcal{P}(\boldsymbol{\alpha})$ for some positive $\epsilon$. Therefore for $i$ in $\mathcal{N}$, the vector $\boldsymbol{\alpha}_{i}$ is expressible as a convex combination of extremal exponents and the zero vector. Let $\boldsymbol{\lambda}^{(i)} \geq 0$ be a vector of convex combination coefficients so that $\boldsymbol{\alpha}_{i}=\sum_{j \in \mathcal{E} \cup\{1\}} \lambda_{j}^{(i)} \boldsymbol{\alpha}_{j}$ with $\lambda_{j}^{(i)}>0$. Now define the vector $\boldsymbol{\nu}^{(i)}$ in $\mathbb{R}^{m}$ by

1. $\nu_{j}^{(i)}=\lambda_{j}^{(i)}$ for $j$ in $\mathcal{E} \cup\{1\}$,

2. $\nu_{j}^{(i)}=-1$ for $j=i$, and

3. $\nu_{j}^{(i)}=0$ for all remaining indices

Each vector $\boldsymbol{\nu}^{(i)}$ satisfies $\nu_{1}^{(i)}>0$, as well as the following constraints associated with $C_{\mathrm{AGE}}(\boldsymbol{\alpha}, i)$ :

$$
\nu_{j}^{(i)} \geq 0 \text { for all } j \neq i \quad \text { and } \quad\left[\begin{array}{c}
\boldsymbol{\alpha} \\
\mathbf{1}^{\top}
\end{array}\right] \boldsymbol{\nu}^{(i)}=\mathbf{0} \in \mathbb{R}^{n+1} .
$$

Because $\nu_{1}^{(i)}$ is positive, the quantity $D\left(\boldsymbol{\nu}_{\backslash i}^{(i)}, \hat{\boldsymbol{c}}_{\backslash i}^{(i)}\right)$ can be made to diverge to $-\infty$ by sending $\gamma$ to $\infty$. It follows that there exists a sufficiently large $M$ so that $\gamma \geq M$ implies

$$
D\left(\boldsymbol{\nu}_{\backslash i}^{(i)}, \hat{\boldsymbol{c}}_{\backslash i}^{(i)}\right)-\left[\sum_{j \neq i} \nu_{j}^{(i)}\right]-\hat{c}_{i} \leq 0 \quad \text { for all } i \text { in } \mathcal{N} .
$$

Equations (11) and (12) tell us that for sufficiently large $\gamma$, we have $\hat{\boldsymbol{c}}^{(i)}$ in $C_{\mathrm{AGE}}(\boldsymbol{\alpha}, i)$ for all $i$ in $\mathcal{N}-$ and the result follows.

\section{Newton Polytopes for SAGE-vs-Nonnegativity}

This section begins by introducing two theorems (Theorems 10 and 11) concerning SAGE representability versus signomial nonnegativity. These theorems are then combined to obtain a third 
theorem (Theorem 12), which provides the most general yet-known conditions for when the SAGE and nonnegativity cones coincide. The proofs of Theorems 10 and 11 are contained in Sections 4.2 and 4.3. Applications of Theorem 10 in the context of signomial optimization are given in Section 4.4 .

\subsection{New Conditions for $C_{\mathrm{SAGE}}(\boldsymbol{\alpha})=C_{\mathrm{NNS}}(\boldsymbol{\alpha})$}

The following theorem is perhaps of greatest practical consequence in this section; it is the first instance beyond AGE functions when SAGE-representability is known to be equivalent to nonnegativity. Its proof (in Section 4.2) makes use of Proposition 1 and subgradient calculus.

Theorem 10. Suppose that ext $\mathcal{P}(\boldsymbol{\alpha})$ is simplicial, and that $\boldsymbol{c}$ has $c_{i} \leq 0$ whenever $\boldsymbol{\alpha}_{i}$ is nonextremal. Then $\boldsymbol{c}$ belongs to $C_{\mathrm{SAGE}}(\boldsymbol{\alpha})$ iff $\boldsymbol{c}$ belongs to $C_{\mathrm{NNS}}(\boldsymbol{\alpha})$.

This section's next theorem (proven in Section 4.3) concerns conditions on $\boldsymbol{\alpha}$ for when the SAGE and nonnegativity cones can be expressed as a Cartesian product of simpler sets. To aid in exposition we introduce a definition: a matrix $\boldsymbol{\alpha}$ can be partitioned into $k$ faces if by a permutation of its columns it can be written as $\boldsymbol{\alpha}=\left[\boldsymbol{\alpha}^{(1)}, \ldots, \boldsymbol{\alpha}^{(k)}\right]$ where $\left\{\mathcal{P}\left(\boldsymbol{\alpha}^{(i)}\right)\right\}_{i=1}^{k}$ are mutually disjoint faces of $\mathcal{P}(\boldsymbol{\alpha})$.

Theorem 11. If $\left\{\boldsymbol{\alpha}^{(i)}\right\}_{i=1}^{k}$ are matrices partitioning $\boldsymbol{\alpha}=\left[\boldsymbol{\alpha}^{(1)}, \ldots, \boldsymbol{\alpha}^{(k)}\right]$, then $C_{\mathrm{NNS}}(\boldsymbol{\alpha})=\oplus_{i=1}^{k} C_{\mathrm{NNS}}\left(\boldsymbol{\alpha}^{(i)}\right)$ and $C_{\mathrm{SAGE}}(\boldsymbol{\alpha})=\oplus_{i=1}^{k} C_{\mathrm{SAGE}}\left(\boldsymbol{\alpha}^{(i)}\right)$.

Here is a simple example of the theorem in action: if every $\boldsymbol{\alpha}_{i}$ is extremal in $\mathcal{P}(\boldsymbol{\alpha})$, then $\boldsymbol{\alpha}$ can be partitioned into $m$ faces and $C_{\mathrm{SAGE}}(\boldsymbol{\alpha})=C_{\mathrm{NNS}}(\boldsymbol{\alpha})=\mathbb{R}_{+}^{m}$. Note that every matrix $\boldsymbol{\alpha}$ admits the trivial partition with $k=1$. In fact a natural regularity condition (one that we consider in Section 6 ) would be that $\boldsymbol{\alpha}$ only admits the trivial partition.

Regularity conditions aside, Theorems 10 and 11 can be combined with known properties of AGE functions to establish new conditions for when the SAGE cone coincides with the nonnegativity cone. Many such conditions of which we are aware are captured in the following theorem.

Theorem 12. Suppose $\boldsymbol{\alpha}$ can be partitioned into faces where (1) each simplicial face contains at most two nonextremal exponents, and (2) all other faces contain at most one nonextremal exponent. Then $C_{\mathrm{SAGE}}(\boldsymbol{\alpha})=C_{\mathrm{NNS}}(\boldsymbol{\alpha})$.

Proof. Let $\boldsymbol{\alpha}$ satisfy the assumptions of Theorem 12 with associated faces $\left\{F_{i}\right\}_{i=1}^{k}$ and column blocks $\boldsymbol{\alpha}^{(i)}$, and fix $\boldsymbol{c}$ in $C_{\mathrm{NNS}}(\boldsymbol{\alpha})$. For $i$ in $[k]$, define the vector $\boldsymbol{c}^{(i)}$ so that $\boldsymbol{c}=\oplus_{i=1}^{k} \boldsymbol{c}^{(i)}$. By Theorem 11, the condition $C_{\mathrm{SAGE}}(\boldsymbol{\alpha})=C_{\mathrm{NNS}}(\boldsymbol{\alpha})$ holds if and only if $C_{\mathrm{SAGE}}\left(\boldsymbol{\alpha}^{(i)}\right)=C_{\mathrm{NNS}}\left(\boldsymbol{\alpha}^{(i)}\right)$ for all $i$ in $[k]$. Because we assumed that $\boldsymbol{c}$ belongs to $C_{\mathrm{NNS}}(\boldsymbol{\alpha})$ it suffices to show that each $\boldsymbol{c}^{(i)}$ belongs to $C_{\mathrm{SAGE}}\left(\boldsymbol{\alpha}^{(i)}\right)$.

First, note that $\boldsymbol{c}^{(i)} \in C_{\mathrm{NNS}}\left(\boldsymbol{\alpha}^{(i)}\right)$ ensures each $\boldsymbol{c}^{(i)}$ contains at most two negative entries. If $\boldsymbol{c}^{(i)}$ has at most one negative entry, then $\boldsymbol{c}^{(i)}$ is simply AGE. If on the other hand $\boldsymbol{c}^{(i)}$ has two negative entries $c_{j}$, then both of these entries must correspond to nonextremal $\boldsymbol{\alpha}_{j}$, and $F_{i}$ must be simplicial. This allows us to invoke Theorem 10 on $\boldsymbol{c}^{(i)}$ to conclude $\boldsymbol{c}^{(i)} \in C_{\mathrm{SAGE}}\left(\boldsymbol{\alpha}^{(i)}\right)$. The result follows.

\subsection{The simplicial sign pattern theorem for SAGE-vs-NNG. (Theorem 10)}

The proof of Theorem 10 begins by exploiting two key facts about signomials and SAGE relaxations: (1) that $C_{\mathrm{SAGE}}(\boldsymbol{\alpha})$ and $C_{\mathrm{NNS}}(\boldsymbol{\alpha})$ are invariant under translation of the exponent set $\boldsymbol{\alpha}$, and (2) that strong duality always holds when computing $f_{\text {SAGE}}$. These properties allow us to reduce the 
problem of checking SAGE decomposability to the problem of exactness of a convex relaxation for a signomial optimization problem. We then take advantage of the relative entropy constraints in the optimization problem to establish sufficient conditions for when the convex relaxation is exact.

Theorem 10. Begin by translating $\boldsymbol{\alpha}$ in $\mathbb{R}^{n \times m}$ to $\boldsymbol{\alpha} \leftarrow \boldsymbol{\alpha}-\boldsymbol{\alpha}_{j} \mathbf{1}^{\top}$ where $\boldsymbol{\alpha}_{j}$ is an arbitrary extremal element of $\mathcal{P}(\boldsymbol{\alpha})$. For ease of exposition we assume that $\mathcal{P}(\boldsymbol{\alpha})$ is full-dimensional. If this is not the case then the proof is easily modified by restricting subsequent discussion to the column space of $\boldsymbol{\alpha}$. With this in mind, permute the columns of $\boldsymbol{\alpha}$ so that $\boldsymbol{\alpha}_{1}=\mathbf{0}$. Fix $\boldsymbol{c}$ in $C_{\mathrm{NNS}}(\boldsymbol{\alpha})$ and define $f=\operatorname{Sig}(\boldsymbol{\alpha}, \boldsymbol{c})$ so that $f^{\star} \geq 0$. We show that $f_{\mathrm{SAGE}}=f^{\star}$, thereby establishing that $\boldsymbol{c}$ belongs to $C_{\text {SAGE }}(\boldsymbol{\alpha})$.

Let $\mathcal{N}=\left\{i: c_{i} \leq 0\right\}$; apply Corollary 3 of Theorem 2 in conjunction with Proposition 1 to obtain

$$
\begin{aligned}
f_{\mathrm{SAGE}}=\inf & \boldsymbol{c}^{\top} \boldsymbol{v} \\
\text { s.t. } & \boldsymbol{v} \text { in } \mathbb{R}_{+}^{m} \text { has } v_{1}=1 \text {, and there exists } \boldsymbol{\mu} \text { in } \mathbb{R}^{n \times m} \text { with } \\
& v_{i} \ln \left(v_{i} / v_{j}\right) \leq\left(\boldsymbol{\alpha}_{i}-\boldsymbol{\alpha}_{j}\right)^{\top} \boldsymbol{\mu}_{i} \text { for } j \text { in }[m] \backslash \mathcal{N} \text { and } i \text { in } \mathcal{N} \cup\{1\} .
\end{aligned}
$$

In order to show $f_{\mathrm{SAGE}}=f^{\star}$, we reformulate (13) as the problem of computing $f^{\star}$ by appropriate changes of variables and constraints.

We begin with a change of constraints. By the assumption that $c_{i} \leq 0$ for all nonextremal $\boldsymbol{\alpha}_{i}$, the index set $\mathcal{E}=[m] \backslash \mathcal{N}$ satisfies $\left\{\boldsymbol{\alpha}_{i}\right\}_{i \in \mathcal{E}} \subset \operatorname{ext} \mathcal{P}(\boldsymbol{\alpha})$. Combine this with the assumption that $\operatorname{ext} \mathcal{P}(\boldsymbol{\alpha})$ is simplicial and the fact that $\mathbf{0}=\boldsymbol{\alpha}_{1}$ is extremal to conclude that $\left\{\boldsymbol{\alpha}_{i}: i\right.$ in $\left.\mathcal{E} \backslash\{1\}\right\}$ are linearly independent. The linear independence of these vectors ensures that for fixed $\boldsymbol{v}$ we can always choose $\boldsymbol{\mu}_{1}$ to satisfy the following constraints with equality

$$
v_{1} \ln \left(v_{1} / v_{j}\right) \leq\left(\boldsymbol{\alpha}_{1}-\boldsymbol{\alpha}_{j}\right)^{\top} \boldsymbol{\mu}_{1} \text { for all } j \text { in }[m] \backslash \mathcal{N} .
$$

Therefore we can equivalently reformulate $f_{\mathrm{SAGE}}$ as

$$
\begin{aligned}
f_{\mathrm{SAGE}}=\inf & \boldsymbol{c}^{\top} \boldsymbol{v} \\
\text { s.t. } & \boldsymbol{v} \text { in } \mathbb{R}_{+}^{m} \text { has } v_{1}=1, \text { and there exists } \boldsymbol{\mu} \text { in } \mathbb{R}^{n \times m} \\
& \text { with } \ln \left(v_{j}\right)=\boldsymbol{\alpha}_{j}^{\top} \boldsymbol{\mu}_{1} \text { for all } j \text { in } \mathcal{E}, \text { and } \\
& v_{i} \ln \left(v_{i} / v_{j}\right) \leq\left(\boldsymbol{\alpha}_{i}-\boldsymbol{\alpha}_{j}\right)^{\top} \boldsymbol{\mu}_{i} \text { for } j \text { in } \mathcal{E}, i \text { in } \mathcal{N} .
\end{aligned}
$$

To reduce the problem further we need to show that for $i$ in $\mathcal{N}$, we can take $v_{i}>0$ without loss of generality. Certainly the objective is decreasing in $v_{i}$, so this boils down to showing that for fixed $\left\{v_{j}\right\}_{j \in \mathcal{E}}$ there exists a choice for $\boldsymbol{\mu}_{i}$ which allows $v_{i}>0$. We need not look far: considering $\boldsymbol{\mu}_{i}=\mathbf{0}$, the choice $v_{i}=\min _{j \in \mathcal{E}} v_{j} / 2$ is always valid.

Having established $\boldsymbol{v}>\mathbf{0}$, the constraint $v_{i} \ln \left(v_{i} / v_{j}\right) \leq\left(\boldsymbol{\alpha}_{i}-\boldsymbol{\alpha}_{j}\right)^{\top} \boldsymbol{\mu}_{i}$ can be rewritten as $\ln \left(v_{i}\right)-\ln \left(v_{j}\right) \leq\left(\boldsymbol{\alpha}_{i}-\boldsymbol{\alpha}_{j}\right)^{\top} \boldsymbol{\mu}_{i}$ by absorbing $v_{i}$ into $\boldsymbol{\mu}_{i}$. If we also substitute the expression for $\ln \left(v_{j}\right)$ given by the equality constraints, then the inequality constraints write as

$$
\ln \left(v_{i}\right) \leq \boldsymbol{\alpha}_{i}^{\top} \boldsymbol{\mu}_{i}+\boldsymbol{\alpha}_{j}^{\top}\left(\boldsymbol{\mu}_{1}-\boldsymbol{\mu}_{i}\right) \text { for all } j \text { in } \mathcal{E}, i \text { in } \mathcal{N} .
$$

Next we show that for every $i$ in $\mathcal{N}$, the choice $\boldsymbol{\mu}_{i}=\boldsymbol{\mu}_{1}$ makes these inequality constraints as loose as possible.

Towards this end, define $\psi_{i}(\boldsymbol{x})=\boldsymbol{\alpha}_{i}^{\top} \boldsymbol{x}+\min _{j \in \mathcal{E}}\left\{\boldsymbol{\alpha}_{j}^{\top}\left(\boldsymbol{\mu}_{1}-\boldsymbol{x}\right)\right\}$; note that for fixed $i$ and $\boldsymbol{\mu}_{i}$, the number $\psi_{i}\left(\boldsymbol{\mu}_{i}\right)$ is the minimum over all $|\mathcal{E}|$ right hand sides in (14). It is easy to verify that $\psi_{i}$ is concave, and because of this we know that $\psi_{i}$ is maximized at $\boldsymbol{x}^{\star}$ if and only if $\mathbf{0} \in\left(\partial \psi_{i}\right)\left(\boldsymbol{x}^{\star}\right)$. 
Standard subgradient calculus tells us that $\left(\partial \psi_{i}\right)(\boldsymbol{x})$ is precisely the convex hull of vectors $\boldsymbol{\alpha}_{i}-\boldsymbol{\alpha}_{k}$ where $k$ is an index at which the minimum (over $j \in \mathcal{E}$ ) is obtained. Therefore $\left(\partial \psi_{i}\right)\left(\boldsymbol{\mu}_{1}\right)=$ $\operatorname{conv}\left\{\boldsymbol{\alpha}_{i}-\boldsymbol{\alpha}_{j}: j\right.$ in $\left.\mathcal{E}\right\}$, and this set must contain the zero vector (unless perhaps $c_{i}=0$, in which case the constraints on $v_{i}$ are inconsequential). Hence $\max _{\boldsymbol{x} \in \mathbb{R}^{n}}\left\{\psi_{i}(\boldsymbol{x})\right\}=\boldsymbol{\alpha}_{i}^{\top} \boldsymbol{\mu}_{1}$, and so inequality constraints (14) reduce to

$$
\ln \left(v_{i}\right) \leq \boldsymbol{\alpha}_{i}^{\top} \boldsymbol{\mu}_{1} \text { for all } i \text { in } \mathcal{N} .
$$

Since the objective $\boldsymbol{c}^{\boldsymbol{\top}} \boldsymbol{v}$ is decreasing in $v_{i}$ for $i$ in $\mathcal{N}$, we can actually take the constraints in (15) to be binding. We established much earlier that $v_{i}=\exp \boldsymbol{\alpha}_{i}^{\top} \boldsymbol{\mu}_{1}$ for $i$ in $\mathcal{E}$. Taking these together we see $v_{i}=\exp \boldsymbol{\alpha}_{i}^{\top} \boldsymbol{\mu}_{1}$ for all $i$, and so

$$
f_{\mathrm{SAGE}}=\inf \left\{\sum_{i=1}^{m} c_{i} \exp \boldsymbol{\alpha}_{i}^{\top} \boldsymbol{\mu}_{1}: \boldsymbol{\mu}_{1} \text { in } \mathbb{R}^{n}\right\}=f^{\star}
$$

as required.

Let us now recap how the assumptions of Theorem 10 were used at various stages in the proof. For one thing, all discussion up to and including the statement of Problem (13) was fully general; the expression for $f_{\mathrm{SAGE}}$ used none of the assumptions of the theorem. The next step was to use linear independence of nonzero extreme points to allow us to satisfy $v_{1} \ln \left(v_{1} / v_{j}\right) \leq\left(\boldsymbol{\alpha}_{1}-\boldsymbol{\alpha}_{j}\right)^{\top} \boldsymbol{\mu}_{1}$ with equality. The reader can verify that if we did not have linear independence but we were told that those constraints were binding at the optimal $\boldsymbol{v}^{\star}$, then we would still have $f_{\mathrm{SAGE}}=f^{\star}$ under the stated sign pattern assumptions on $\boldsymbol{c}$.

Our first use of the sign pattern assumptions on $c$ was quite modest; it was only to justify $v_{i}>0$ and in turn to redefine $\boldsymbol{\mu}_{i}$ so that $v_{i} \ln \left(v_{i} / v_{j}\right) \leq\left(\boldsymbol{\alpha}_{i}-\boldsymbol{\alpha}_{j}\right)^{\top} \boldsymbol{\mu}_{i}$ could be rewritten as in (14). The discussion that immediately followed (i.e. replacing (14) by (15)) didn't actually use the sign pattern assumptions at all; it simply chose $\boldsymbol{\mu}_{i}$ to make the constraints in (14) as loose as possible. It was only in replacing (15) by $\ln \left(v_{i}\right)=\boldsymbol{\alpha}_{i}^{\top} \boldsymbol{\mu}_{1}$ that we relied fully on the sign pattern assumptions on $\boldsymbol{c}$.

\subsection{Proof of the partitioning theorem (Theorem 11)}

This section's first lemma adapts claim (iv) from Theorem 3.6 of Reznick [36] to signomials. Because the lemma is important for our subsequent theorems, we provide a more complete proof than can be found in Reznick's [36]. As a matter of notation: for any $A \subset \mathbb{R}^{n}$, write $\operatorname{Sig}_{A}(\boldsymbol{\alpha}, \boldsymbol{c})$ to mean the signomial with exponents $\boldsymbol{\alpha}_{i}$ in $A$ and corresponding coefficients $c_{i}$.

Lemma 13. Fix $f=\operatorname{Sig}(\boldsymbol{\alpha}, \boldsymbol{c})$. If $F$ is a face of $\mathcal{P}(\boldsymbol{\alpha})$ and $g=\operatorname{Sig}_{F}(\boldsymbol{\alpha}, \boldsymbol{c})$, then $g^{\star}<0$ implies $f^{\star}<0$.

Proof. For brevity write $P=\mathcal{P}(\boldsymbol{\alpha})$; we may assume without loss of generality that $P$ contains the origin. If $F=P$ then $g=f$ and the claim is trivial. If otherwise, the affine hull of $F$ must have some positive codimension $\ell$, and there exist supporting hyperplanes $\left\{S_{i}\right\}_{i=1}^{\ell}$ such that $F=\left[\cap_{i=1}^{\ell} S_{i}\right] \cap P$. We can express $S_{i}$ as $\left\{\boldsymbol{x}: \boldsymbol{s}_{i}^{\top} \boldsymbol{x}=r_{i}\right\}$ for a vector $\boldsymbol{s}_{i}$ and a scalar $r_{i}$. Because $P$ is convex we know that it is contained in one of the half spaces $\left\{\boldsymbol{x}: \boldsymbol{s}_{i}^{\top} \boldsymbol{x} \leq r_{i}\right\}$ or $\left\{\boldsymbol{x}: \boldsymbol{s}_{i}^{\top} \boldsymbol{x} \geq r_{i}\right\}$. By possibly replacing $\left(\boldsymbol{s}_{i}, r_{i}\right)$ by $\left(-\boldsymbol{s}_{i},-r_{i}\right)$, we can assume that $P$ is contained in $\left\{\boldsymbol{x}: \boldsymbol{s}_{i}^{\top} \boldsymbol{x} \leq r_{i}\right\}$. In addition, the assumption that $\mathbf{0}$ belongs to $P$ ensures that each $r_{i}$ is nonnegative. Now define $s=\sum_{i=1}^{\ell} \boldsymbol{s}_{i}$ and $r=\sum_{i=1}^{\ell} r_{i} \geq 0$. The pair $(s, r)$ is constructed to satisfy the following properties:

- For every $\boldsymbol{\alpha}_{j}$ in $F$, we have $\boldsymbol{\alpha}_{j}^{\top} s=r$.

- For every $\boldsymbol{\alpha}_{j}$ not in $F$, we have $\boldsymbol{\alpha}_{j}^{\top} s<r$. 
Finally, define $h=\operatorname{Sig}_{P \backslash F}(\boldsymbol{\alpha}, \boldsymbol{c})$ so that $f=g+h$. The remainder of the proof is case analysis on $r$.

If $r=0$ then we must have $r_{i}=0$ for all $i$. The condition that $r_{i}=0$ for all $i$ implies that $F$ is contained in a linear subspace $U$ which is orthogonal to $s$, and so nonnegativity of $g$ over $\mathbb{R}^{n}$ reduces to nonnegativity of $g$ over $U$. Suppose then that there exists some $\hat{\boldsymbol{x}}$ in $U$ where $g(\hat{\boldsymbol{x}})$ is negative. For any vector $\boldsymbol{y}$ in the orthogonal complement of $U$ we have $g(\hat{\boldsymbol{x}}+\boldsymbol{y})=g(\hat{\boldsymbol{x}})$. Meanwhile no matter the value of $\hat{\boldsymbol{x}}$ we know that $\lim _{t \rightarrow \infty} h(\hat{\boldsymbol{x}}+t \boldsymbol{s})=0$. Using $f^{\star} \leq \inf \{f(\hat{\boldsymbol{x}}+t \boldsymbol{s}): t$ in $\mathbb{R}\} \leq g(\hat{\boldsymbol{x}})$, we have the desired result for $r=0: g^{\star}<0$ implies $f^{\star}<0$.

Now consider the case when $r$ is positive. Define the vector $\hat{s}=r \boldsymbol{s} /\|\boldsymbol{s}\|^{2}$; we produce an upper bound on $f^{\star}$ by searching over all hyperplanes $\left\{\boldsymbol{x}: \hat{\boldsymbol{s}}^{\top} \boldsymbol{x}=t\right\}$ for $t$ in $\mathbb{R}$. Specifically, for any $\boldsymbol{x}$ in $\mathbb{R}^{n}$ there exists a scalar $t$ and a vector $\boldsymbol{y}$ such that $\boldsymbol{x}=t \hat{\boldsymbol{s}}+\boldsymbol{y}$ and $\hat{\boldsymbol{s}}^{\top} \boldsymbol{y}=0$. In these terms we have

$$
g(t \hat{\boldsymbol{s}}+\boldsymbol{y})=\exp \left(t\|\hat{\boldsymbol{s}}\|^{2}\right) \sum_{\boldsymbol{\alpha}_{i} \in F} c_{i} \underbrace{\exp \left(t\left[\boldsymbol{\alpha}_{i}-\hat{\boldsymbol{s}}\right]^{\top} \hat{\boldsymbol{s}}\right)}_{=1 \text { for all } t} \exp \left(\boldsymbol{\alpha}_{i}^{\top} \boldsymbol{y}\right) .
$$

Hence our assumption that $g^{\star}<0$ implies the existence of some $\hat{\boldsymbol{y}}$ in $\operatorname{Span}(\boldsymbol{s})^{\perp}$ where $\sum_{\boldsymbol{\alpha}_{i} \in F} c_{i} \exp \left(\boldsymbol{\alpha}_{i}^{\top} \hat{\boldsymbol{y}}\right)$ is negative. Using this $\hat{\boldsymbol{y}}$, one may verify that

$$
\lim _{t \rightarrow \infty} f(t \hat{\boldsymbol{s}}+\hat{\boldsymbol{y}})=-\infty
$$

and so when $r$ is positive, $g^{\star}<0$ implies $f^{\star}<0$.

Theorem 11. Let $\boldsymbol{\alpha}$ have partition $\boldsymbol{\alpha}=\left[\boldsymbol{\alpha}^{(1)}, \ldots, \boldsymbol{\alpha}^{(k)}\right]$. It is clear from the definition of the SAGE cone that $C_{\mathrm{SAGE}}(\boldsymbol{\alpha})=\oplus_{i=1}^{k} C_{\mathrm{SAGE}}\left(\boldsymbol{\alpha}^{(i)}\right)$. The bulk of this proof is to show that $C_{\mathrm{NNS}}(\boldsymbol{\alpha})$ admits the same decomposition.

Let $f=\operatorname{Sig}(\boldsymbol{\alpha}, \boldsymbol{c})$ for some $\boldsymbol{c}$ in $\mathbb{R}^{n}$. The vector $\boldsymbol{c}$ is naturally decomposed into $\boldsymbol{c}=\oplus_{i=1}^{k} \boldsymbol{c}^{(i)}$ in the same way as $\boldsymbol{\alpha}$ is decomposed into $\boldsymbol{\alpha}^{(i)}$. For each $i$ in $[k]$ define $f_{i}=\operatorname{Sig}\left(\boldsymbol{\alpha}^{(i)}, \boldsymbol{c}^{(i)}\right)$ so that $f=\sum_{i=1}^{k} f_{i}$. If any $f_{i}^{\star}$ is negative, then Lemma 13 tells us that $f^{\star}$ must also be negative. Meanwhile if all $f_{i}^{\star}$ are nonnegative, then the same must be true of $f^{\star} \geq \sum_{i=1}^{k} f_{i}^{\star}$. The result follows.

Although Theorem 11 is stated in terms of nonnegativity, it has a natural optimization variant when $\boldsymbol{\alpha}_{1}=\mathbf{0}$ : If $\left\{F_{i}\right\}_{i=1}^{k}$ partitions the matrix of exponent vectors $\boldsymbol{\alpha}$, then $f^{\star}=\min _{i \in[k]} \operatorname{Sig}_{F_{i}}(\boldsymbol{\alpha}, \boldsymbol{c})^{\star}$.

\subsection{Corollaries for signomial programming}

Signomial minimization is naturally related via duality to checking signomial nonnegativity. Thus we build on groundwork laid in Section 3 and earlier in Section 4 to obtain consequences for signomial minimization.

Corollary 14. Assume that $\mathcal{P}(\boldsymbol{\alpha})$ is simplicial, and that nonzero nonextremal $\boldsymbol{\alpha}_{i}$ have $c_{i} \leq 0$. Then either $f_{\mathrm{SAGE}}=f^{\star}$, or $f^{\star} \in\left(f_{\mathrm{SAGE}}, c_{1}\right)$.

Proof. It suffices to show that $f_{\mathrm{SAGE}}<f^{\star}$ implies $f^{\star}<c_{1}$. This follows as the contrapositive of the following statement: "If $f^{\star} \geq c_{1}$, then by Theorem 10 the nonnegative signomial $f-f^{\star}$ is SAGE, which in turn ensures $f_{\mathrm{SAGE}}=f^{\star}$."

Now we consider constrained signomial programs. Starting with problem data $(f, g)$ where $f=\operatorname{Sig}(\boldsymbol{\alpha}, \boldsymbol{c})$ and $g_{j}=\operatorname{Sig}\left(\boldsymbol{\alpha}, \boldsymbol{g}_{j}\right)$ for $j$ in $[k]$, consider the problem of computing

$$
(f, g)^{\star}=\inf \left\{f(\boldsymbol{x}): \boldsymbol{x} \text { in } \mathbb{R}^{n} \text { satisfies } g(\boldsymbol{x}) \geq \mathbf{0}\right\} .
$$


It is evident ${ }^{2}$ that we can relax the problem to that of computing

$$
(f, g)_{\mathrm{SAGE}} \doteq \inf \left\{\boldsymbol{c}^{\boldsymbol{\top}} \boldsymbol{v}: \boldsymbol{v} \text { in } C_{\mathrm{SAGE}}(\boldsymbol{\alpha})^{\dagger} \text { satisfies } v_{1}=1 \text { and } \boldsymbol{G}^{\boldsymbol{\top}} \boldsymbol{v} \geq \mathbf{0}\right\}
$$

where $\boldsymbol{G}$ is the $m \times k$ matrix whose columns are the $\boldsymbol{g}_{j}$.

Corollary 15. Suppose that $\mathcal{P}(\boldsymbol{\alpha})$ is simplicial with vertex $\boldsymbol{\alpha}_{1}=\mathbf{0}$, and that when $\boldsymbol{\alpha}_{i}$ is nonextremal we have that

1. $\boldsymbol{c}^{\boldsymbol{\top}} \boldsymbol{v}$ is decreasing in $v_{i}$, and

2. each $\boldsymbol{g}_{j}^{\top} \boldsymbol{v}$ is increasing in $v_{i}$.

Then $(f, g)_{\mathrm{SAGE}}=(f, g)^{\star}$.

sketch. The claim that $(f, g)_{\mathrm{SAGE}}=(f, g)^{\star}$ can be established by a change-of-variables and changeof-constraints argument of the same kind used in the proof of Theorem 10.

Suffice it to say that rather than using Theorem 2 and Proposition 1 to justify removing constraints from the dual without loss of generality, one can simply throw out those constraints to obtain some $(f, g)^{\prime}$ with $(f, g)^{\prime} \leq(f, g)_{\text {SAGE }}$. One then shows $(f, g)^{\prime}=(f, g)^{\star}$ to sandwich $(f, g)^{\star} \leq(f, g)^{\prime} \leq(f, g)_{\mathrm{SAGE}} \leq(f, g)^{\star}$.

\section{Certifying Global Nonnegativity of Polynomials}

Let $\boldsymbol{M}_{n, d}$ denote the $n$-by- $\left(\begin{array}{c}n+d \\ d\end{array}\right)$ matrix whose columns are vectors in the nonnegative integer lattice with entries summing to at most $d$. Historically the cone of nonnegative polynomials has been studied as

$$
\Sigma_{n, d}=\left\{\boldsymbol{c}: \operatorname{Poly}\left(\boldsymbol{M}_{n, d}, \boldsymbol{c}\right)(\boldsymbol{x}) \geq 0 \text { for all } \boldsymbol{x} \text { in } \mathbb{R}^{n}\right\} .
$$

This article takes a different perspective, one informed by our interest in sparse polynomials and nonnegativity certificates with complexity not depending on the polynomial's degree. Specifically, to any $n$-by- $m$ matrix of nonnegative integers $\boldsymbol{\alpha}$, we associate the cone

$$
C_{\mathrm{NNP}}(\boldsymbol{\alpha}) \doteq\left\{\boldsymbol{c}: \operatorname{Poly}(\boldsymbol{\alpha}, \boldsymbol{c})(\boldsymbol{x}) \geq 0 \text { for all } \boldsymbol{x} \text { in } \mathbb{R}^{n}\right\}
$$

The goal of this section is to derive and analyze a tractable relative-entropy-representable inner approximation of $C_{\mathrm{NNP}}(\boldsymbol{\alpha})$. At the core of the derivation is a method for the following task: Given a polynomial $p$, obtain a signomial $q$ where nonnegativity of $q$ certifies nonnegativity of $p$. We discuss how to accomplish this task in Section 5.1. Once this is done, Section 5.2 defines and subsequently analyzes the notion of $S A G E$ polynomials - polynomials that are globally nonnegative and certifiably so via a small number of relative entropy inequalities. Section 5.3 addresses the merits of our approach over existing methods such as SONC, SDSOS, and SOS. Section 5.4 concludes with an outline of how to use SAGE polynomials to obtain a hierarchy for constrained polynomial optimization.

\footnotetext{
${ }^{2}$ See Section 3.4 of [9].
} 


\subsection{Orthant selection and signomial representatives}

Here we address the fundamental problem of obtaining a signomial $q$ from a polynomial $p$ in such a way that nonnegativity of $q$ implies nonnegativity of $p$. One way to do this is by defining $q(\boldsymbol{x})=p(\exp \boldsymbol{x}-\exp (-\boldsymbol{x}))$. This transformation gets the job done, in that such $q$ would be nonnegative if and only if $p$ was nonnegative. The trouble with this approach is that a monomial $\boldsymbol{x}^{\boldsymbol{\alpha}_{i}}$ from $p$ would contribute a number of terms to $q$ on the order of the multinomial coefficient $\left(\begin{array}{c}\boldsymbol{\alpha}_{i}^{\top} \mathbf{1} \\ \boldsymbol{\alpha}_{i}\end{array}\right)$ - and this can be exponentially large in the monomial degree $\boldsymbol{\alpha}_{i}^{\top} \mathbf{1}$.

Consider instead an orthant-by-orthant approach. Every orthant in $\mathbb{R}^{n}$ is canonically associated with a vertex of the hypercube $\{ \pm 1\}^{n}$ by making use of the Hadamard product. First we extend the Hadamard product to support sets in one argument by $\boldsymbol{a} \odot B \doteq\{\boldsymbol{a} \odot \boldsymbol{b}: \boldsymbol{b}$ in $B\}$, and then make the identification $\mathbb{R}_{s}^{n} \doteq \boldsymbol{s} \odot \mathbb{R}_{+}^{n}$. Thus while $q(\boldsymbol{x})=p(\exp \boldsymbol{x})$ is nonnegative over $\mathbb{R}^{n}$ iff $p$ is nonnegative over $\mathbb{R}_{+}^{n}$, the signomial $q_{\boldsymbol{s}}(\boldsymbol{x}) \doteq p(\boldsymbol{s} \odot \exp \boldsymbol{x})$ is nonnegative over $\mathbb{R}^{n}$ iff $p$ is nonnegative over $\mathbb{R}_{\boldsymbol{s}}^{n}$. This view of constructing signomial representatives is sparsity-preserving and degree independent; the task of determining which orthants require a signomial representative shall be called "orthant selection."

The simplest way to manage orthant selection is to require that all exponent vectors $\boldsymbol{\alpha}_{i}$ belong to the even integer lattice, thereby rendering all orthants equivalent (c.f. [21]). However we are interested in making less restrictive assumptions. Towards this end, consider how orthants in $\mathbb{R}^{n}$ are naturally related to orthants in $\mathbb{R}^{m}$ by the correspondence $s \mapsto s^{\alpha}$ where $\boldsymbol{t}=\boldsymbol{s}^{\alpha}$ has values $t_{i}=\boldsymbol{s}^{\boldsymbol{\alpha}_{i}}$. This correspondence is such that $\operatorname{Sig}(\boldsymbol{\alpha}, \boldsymbol{c} \odot \boldsymbol{t})$ is nonnegative over $\mathbb{R}^{n}$ if and only if $p$ is nonnegative over $\mathbb{R}_{s}^{n}$. In view of this we ask if there exists a distinguished orthant $s$ where

$$
\left\{i: c_{i} \cdot \boldsymbol{s}^{\boldsymbol{\alpha}_{i}}<0\right\} \text { contains }\left\{i: c_{i} \cdot \hat{\boldsymbol{s}}^{\boldsymbol{\alpha}_{i}}<0\right\} \text { for all } \hat{\boldsymbol{s}} \text { in }\{ \pm 1\}^{n} \text {. }
$$

If such an orthant exists, then global nonnegativity of $p$ would follow from nonnegativity over $\mathbb{R}_{s}^{n}$. Assuming $s$ specifies such an orthant, the choice $\boldsymbol{t}=\boldsymbol{s}^{\boldsymbol{\alpha}}$ would have $q_{\boldsymbol{t}} \doteq \operatorname{Sig}(\boldsymbol{\alpha}, \boldsymbol{c} \odot \boldsymbol{t})$ nonnegative iff $p$ were nonnegative.

This structure allows us to move between polynomials and signomials without loss of generality. Moving forward, if $p=\operatorname{Poly}(\boldsymbol{\alpha}, \boldsymbol{c})$ is such that (20) holds for some $\boldsymbol{s}$ in $\{ \pm 1\}^{n}$, then $p$ shall be called a single-orthant dominated polynomial, and $s$ will be called its distinguished orthant. Verifying that a polynomial is single-orthant dominated is a very simple computational task. Given $\boldsymbol{\alpha}$ and $\boldsymbol{c}$, define $\boldsymbol{b}$ by

$$
b_{i}=\left\{\begin{array}{ll}
0 & \text { if } c_{i} \leq 0 \text { or } \boldsymbol{\alpha}_{i} \text { even } \\
1 & \text { if otherwise }
\end{array} .\right.
$$

Then assuming every $c_{i} \neq 0$, the polynomial $p=\operatorname{Poly}(\boldsymbol{\alpha}, \boldsymbol{c})$ is single-orthant dominated if and only if the system

$$
\boldsymbol{\alpha}^{\top} \boldsymbol{x}=\boldsymbol{b} \quad(\bmod 2)
$$

has a solution in $\boldsymbol{x}$ over $\mathbb{F}_{2}^{n}$. Moreover, if such $\boldsymbol{x}$ exists, then the distinguished orthant for $p$ is given by $s$ with $s_{i}=-1$ when $x_{i}=1$, and $s_{i}=1$ when $x_{i}=0$.

The system (21) can be solved (or proven inconsistent) in $O\left(m n^{2}\right)$ time by applying Gaussian elimination over the finite field $\mathbb{F}_{2}$. If one desires a certificate that a polynomial is single-orthant dominated, then solving (21) to recover the distinguished orthant $s$ is indeed recommended. However, if we are willing to assume that $p$ is single-orthant dominated, we can simply choose $\boldsymbol{t}$ in $\{ \pm 1\}^{m}$ with

$$
t_{i}=\left\{\begin{aligned}
1 & \text { if } c_{i} \leq 0 \text { or } \boldsymbol{\alpha}_{i} \text { even } \\
-1 & \text { if otherwise }
\end{aligned}\right.
$$


In fact, we can simplify the bookkeeping even further by noting that $\hat{\boldsymbol{c}} \doteq \boldsymbol{c} \odot \boldsymbol{t}$ satisfies

$$
\hat{c}_{i}=\left\{\begin{array}{cl}
c_{i} & \text { if } \boldsymbol{\alpha}_{i} \text { even } \\
-\left|c_{i}\right| & \text { if otherwise }
\end{array} .\right.
$$

As the description of $\hat{\boldsymbol{c}}$ does not ultimately depend on the choice of $\boldsymbol{t}$, this leads us to the natural conclusion for managing orthant selection: regardless of whether or not $p$ is single-orthant dominated, define $\hat{\boldsymbol{c}}$ by (23), and construct the "signomial representative" $q=\operatorname{Sig}(\boldsymbol{\alpha}, \hat{\boldsymbol{c}})$. Nonnegativity of the signomial representative is always a sufficient condition for nonnegativity of $p$; in the case of single-orthant dominated polynomials, nonnegativity of the signomial representative is also necessary.

\subsection{SAGE polynomials}

We say that $p=\operatorname{Poly}(\boldsymbol{\alpha}, \boldsymbol{c})$ is a $S A G E$ polynomial if its signomial representative $q=\operatorname{Sig}(\boldsymbol{\alpha}, \hat{\boldsymbol{c}})(\hat{\boldsymbol{c}}$ given by (23)) is a SAGE signomial. By construction, SAGE polynomials are nonnegative over the whole of $\mathbb{R}^{n}$.

As with SAGE signomials, we can define the cone of valid coefficients with respect to a matrix of fixed exponent vectors $\boldsymbol{\alpha}$. Specifically, we define

$$
\begin{aligned}
C_{\mathrm{SAGE}}^{\mathrm{POLY}}(\boldsymbol{\alpha}) & \doteq\{\boldsymbol{c}: \operatorname{Poly}(\boldsymbol{\alpha}, \boldsymbol{c}) \text { is a SAGE polynomial }\} \\
& =\left\{\boldsymbol{c}: \hat{\boldsymbol{c}} \text { in } C_{\mathrm{SAGE}}(\boldsymbol{\alpha}), \hat{\boldsymbol{c}} \text { is given by }(23)\right\}
\end{aligned}
$$

It is desirable to express " $c \in C_{\mathrm{SAGE}}^{\mathrm{POLY}}(\boldsymbol{\alpha})$ " by constraints that are convex in $\boldsymbol{c}$. At first glance, the nonconvex equality constraints between $\boldsymbol{c}$ and $\hat{\boldsymbol{c}}$ in (23) seem to pose a problem. However, one can readily verify that the problematic equality constraints $\hat{c}_{i}=-\left|c_{i}\right|$ can be relaxed to the convex inequality constraints $\hat{c}_{i} \leq-\left|c_{i}\right|$ without loss of generality. ${ }^{3}$ Hence we obtain the convex description

$$
\begin{aligned}
C_{\mathrm{SAGE}}^{\mathrm{POLY}}(\boldsymbol{\alpha})=\{\boldsymbol{c}: & \text { there exists } \hat{\boldsymbol{c}} \text { in } C_{\mathrm{SAGE}}(\boldsymbol{\alpha}) \text { satisfying } \\
& \hat{c}_{i}=c_{i} \text { for all } i \text { with } \boldsymbol{\alpha}_{i} \text { in }(2 \mathbb{N})^{n}, \text { and } \\
& \left.\hat{c}_{i} \leq-\left|c_{i}\right| \text { for all } i \text { with } \boldsymbol{\alpha}_{i} \text { not in }(2 \mathbb{N})^{n}\right\} .
\end{aligned}
$$

Equation (26) should leave no question regarding the tractability of optimizing over $C_{\mathrm{SAGE}}^{\mathrm{POLY}}(\boldsymbol{\alpha})$. Given this, we turn to extending our results for signomial nonnegativity to the realm of polynomial nonnegativity. Our first such result is a direct consequence of Theorem 10.

Corollary 16. Let $p$ be a polynomial with (1) simplicial Newton polytope, and (2) the property that its nonextremal exponent vectors are linearly independent mod 2. Then $p$ is nonnegative if and only if it is $S A G E$.

Note that the linear independence of nonextremal exponent vectors effectively requires that any even exponent vector is extremal. We can simultaneously make this assumption more explicit, as well as have a more general result by making reference to single-orthant dominated polynomials.

Corollary 17. Let $p=\operatorname{Poly}(\boldsymbol{\alpha}, \boldsymbol{c})$ be a single-orthant dominated polynomial with simplicial Newton polytope. Suppose that every $\boldsymbol{\alpha}_{i}$ that is even and has positive coefficient is extremal. Then $p$ is nonnegative iff it is SAGE.

And of course, we can extend Theorem 12 to the polynomial case.

\footnotetext{
${ }^{3}$ In practice, this would be written as two linear constraints $\hat{c}_{i} \leq c_{i}$ and $\hat{c}_{i} \leq-c_{i}$.
} 
Corollary 18. Suppose $\boldsymbol{\alpha}$ belonging to $p=\operatorname{Poly}(\boldsymbol{\alpha}, \boldsymbol{c})$ can be partitioned into faces where (1) each simplicial face induces a single-orthant dominated polynomial with at most two nonextremal exponents, and (2) all other faces contain at most one nonextremal exponent. Then $p$ is nonnegative iff it is $S A G E$.

Now we use the results from Section 3 to better understand the cone of SAGE polynomials. Although $C_{\mathrm{SAGE}}^{\mathrm{POLY}}(\boldsymbol{\alpha})$ can be given by any of Equations (24) through (26), it is helpful to reverse engineer these definitions to arrive at the idea of an AGE polynomial. The following lemma presents the result of this reverse-engineering.

Lemma 19. Define the cone of $A G E$ polynomials with respect to exponents $\boldsymbol{\alpha}$ and index $k$ as

$$
\begin{aligned}
C_{\mathrm{AGE}}^{\mathrm{POLY}}(\boldsymbol{\alpha}, k) \doteq\{\boldsymbol{c}: & \text { Poly }(\boldsymbol{\alpha}, \boldsymbol{c}) \text { globally nonnegative, and } \\
& \left.\boldsymbol{c}_{\backslash k} \geq \mathbf{0}, c_{i}=0 \text { for all } i \neq k \text { with } \boldsymbol{\alpha}_{i} \notin(2 \mathbb{N})^{n}\right\} .
\end{aligned}
$$

Then

$$
C_{\mathrm{SAGE}}^{\mathrm{POLY}}(\boldsymbol{\alpha})=\sum_{\boldsymbol{\alpha} \boldsymbol{k} \notin \operatorname{ext} \mathcal{P}(\boldsymbol{\alpha})} C_{\mathrm{AGE}}^{\mathrm{POLY}}(\boldsymbol{\alpha}, k)
$$

where $C_{\mathrm{SAGE}}^{\mathrm{POLY}}(\boldsymbol{\alpha})$ can be defined by any of Equations (24) through (26).

Proof. Given a polynomial $p=\operatorname{Poly}(\boldsymbol{\alpha}, \boldsymbol{c})$, testing if $\boldsymbol{c}$ belongs to $C_{\mathrm{SAGE}}^{\mathrm{POLY}}(\boldsymbol{\alpha})$ will reduce to testing if $\hat{\boldsymbol{c}}$ (given by Equation (23)) belongs to $C_{\mathrm{SAGE}}(\boldsymbol{\alpha})$. The vector $\hat{\boldsymbol{c}}$ will be decomposed to $\hat{\boldsymbol{c}}=\sum_{i} \hat{\boldsymbol{c}}^{(i)}$ where each $\hat{\boldsymbol{c}}^{(i)}$ belongs to $C_{\mathrm{AGE}}(\boldsymbol{\alpha}, i)$. An arbitrary vector $\hat{\boldsymbol{c}}^{(i)}$ in $C_{\mathrm{AGE}}(\boldsymbol{\alpha}, i)$ does not in-of-itself certify nonnegativity of a polynomial $p_{i}=\operatorname{Poly}\left(\boldsymbol{\alpha}, \hat{\boldsymbol{c}}^{(i)}\right)$. One simple example of this is that if $\hat{\boldsymbol{c}}^{(i)} \geq \mathbf{0}$, then $p_{i}$ could easily be unbounded below if some extremal exponent vector $\boldsymbol{\alpha}_{k}$ was not even, and had positive $\hat{c}_{k}$. To get around this, we need to use Theorem 2 .

Let $\mathcal{N}=\left\{i: \hat{c}_{i}<0\right\}$. Then by Theorem 2, $\hat{\boldsymbol{c}}$ belongs to $C_{\mathrm{SAGE}}(\boldsymbol{\alpha})$ if and only if there exist vectors $\left\{\hat{\boldsymbol{c}}^{(i)}\right\}_{i \in \mathcal{N}}$ where for every index $i \in \mathcal{N}$

1. the vector $\hat{\boldsymbol{c}}^{(i)}$ belongs to $C_{\mathrm{AGE}}(\boldsymbol{\alpha}, i)$,

2. $\hat{c}_{i}^{(i)}=\hat{c}_{i}<0$, and

3. $\hat{c}_{j}^{(i)}=0$ for all $j$ in $\mathcal{N} \backslash\{i\}$.

The sign patterns here are important: $\hat{\boldsymbol{c}}^{(i)}$ is supported on the index set $\{i\} \cup([m] \backslash \mathcal{N})$, and $\hat{c}_{j}^{(i)} \geq 0$ for all $j$ in $[m] \backslash \mathcal{N}$. By construction of $\hat{\boldsymbol{c}}$, any index $j$ in $[m] \backslash \mathcal{N}$ corresponds to an exponent vector $\boldsymbol{\alpha}_{j}$ in $(2 \mathbb{N})^{n}$. Therefore the carefully chosen vectors $\left\{\hat{\boldsymbol{c}}^{(i)}\right\}_{i \in \mathcal{N}}$ define not only nonnegative signomials, but also nonnegative polynomials $p_{i}=\operatorname{Poly}\left(\boldsymbol{\alpha}, \hat{\boldsymbol{c}}^{(i)}\right)$.

Expressing the cone of SAGE polynomials in this way is instrumental next in characterizing its extreme rays.

Theorem 20. The extreme rays of the cone $C_{\mathrm{SAGE}}^{\mathrm{POLY}}(\boldsymbol{\alpha})$ are supported on either a single coordinate, or a set of coordinates inducing a simplicial circuit.

Proof. In view of Lemma 19, it suffices to show that for fixed $k$ the extreme rays of $C_{\mathrm{AGE}}^{\mathrm{POLY}}(\boldsymbol{\alpha}, k)$ are supported on single coordinates, or simplicial circuits. Henceforth, let $\boldsymbol{c}$ be a vector along an extreme ray of $C_{\mathrm{AGE}}^{\mathrm{POLY}}(\boldsymbol{\alpha}, k)$. Of course, if $c_{k}=0$, then $\boldsymbol{c}$ can only be supported on single index $i \neq k$. The interesting case is when $c_{k} \neq 0$. 
To $\boldsymbol{c}$ associate $\hat{\boldsymbol{c}}$ as given by $\hat{\boldsymbol{c}}_{\backslash k}=\boldsymbol{c}_{\backslash k}$ and $\hat{c}_{k}=-\left|c_{k}\right|$. By the definition of the cone $C_{\mathrm{AGE}}^{\mathrm{POLY}}(\boldsymbol{\alpha}, k)$, the vector $\hat{\boldsymbol{c}}$ belongs to $C_{\mathrm{AGE}}(\boldsymbol{\alpha}, k)$. Furthermore, it is obvious that $\hat{\boldsymbol{c}}$ has the same support as $\boldsymbol{c}$. Since $\hat{c}_{k}$ is negative and $\hat{\boldsymbol{c}}$ belongs to $C_{\mathrm{AGE}}(\boldsymbol{\alpha}, k)$, it follows that $\hat{\boldsymbol{c}}$ cannot be supported on a single coordinate. Therefore if we can show that $\hat{\boldsymbol{c}}$ lays along an extreme ray of $C_{\mathrm{AGE}}(\boldsymbol{\alpha}, k)$, then both $\hat{\boldsymbol{c}}$ and $\boldsymbol{c}$ must be supported over exponent vectors defining a simplicial circuit.

The proof that $\hat{\boldsymbol{c}}$ is extremal is by contradiction. To begin, suppose $\hat{\boldsymbol{c}}$ can be written as $\hat{\boldsymbol{c}}=\hat{\boldsymbol{c}}^{(1)}+\hat{\boldsymbol{c}}^{(2)}$ with $\hat{\boldsymbol{c}}^{(1)}, \hat{\boldsymbol{c}}^{(2)}$ in $C_{\mathrm{AGE}}(\boldsymbol{\alpha}, k)$ and $\hat{\boldsymbol{c}}^{(1)} \not \alpha \hat{\boldsymbol{c}}^{(2)}$. We will use the AGE signomial vectors $\hat{\boldsymbol{c}}^{(i)}$ to back out a similar decomposition of $\boldsymbol{c}$ in terms of AGE polynomial vectors $\boldsymbol{c}^{(i)}$. As a first step, note that $\hat{\boldsymbol{c}} ¥ \mathbf{0}$ ensures that at least one of $\hat{c}_{k}^{(i)}$ is negative. By relabeling we may assume this is $i=1$. But what's more: by possibly reassigning the $\hat{\boldsymbol{c}}^{(i)}$ 's with appropriate convex combinations of one another, we can be certain that $\hat{c}_{k}^{(2)} \leq 0$ while retaining $\hat{\boldsymbol{c}}^{(1)} \not \subset \hat{\boldsymbol{c}}^{(2)}{ }^{4}$ Now using $\hat{c}_{k}^{(2)} \leq 0$, it is easily seen that $\hat{\boldsymbol{c}}^{(1)}$ and $\hat{\boldsymbol{c}}^{(2)}$ define AGE polynomials. As a last step, define another pair of AGE polynomial vectors $\boldsymbol{c}^{(i)}$ by $\boldsymbol{c}_{\backslash k}^{(i)}=\hat{\boldsymbol{c}}_{\backslash k}^{(i)}$, and $c_{k}^{(i)}=-1 \cdot \operatorname{sign}\left(\hat{c}_{k}\right) \cdot \hat{c}_{k}^{(i)}$. These $\boldsymbol{c}^{(i)}$ inherit nonproportionality from the $\hat{\boldsymbol{c}}^{(i)}$ 's, and they sum to $\boldsymbol{c}$. However, this decomposition of $\boldsymbol{c}$ violates our assumption that $\boldsymbol{c}$ was extremal, and so we have arrived at a contradiction! Our assumption that $\hat{\boldsymbol{c}}$ was nonextremal was false.

We have shown that the vector $\hat{\boldsymbol{c}}$ is extremal in $C_{\mathrm{SAGE}}(\boldsymbol{\alpha})$. Furthermore, $\hat{c}_{k}<0$ ensures that $\hat{\boldsymbol{c}}$ does not define a trivial extreme ray, and so by Theorem 4 it must be supported on a simplicial circuit. As $\hat{\boldsymbol{c}}$ and $\boldsymbol{c}$ have the same support- our proof is complete.

\subsection{Comparison to other polynomial nonnegativity certificates}

In this section we address how sums-of-squares (SOS) [33, 27, 42], scaled-diagonally-dominant-sumsof-squares (SDSOS) [2, 3], and sums-of-nonnegative-circuit-polynomials (SONC) [19, 20, 12, 38] compare to the method of SAGE polynomials.

\subsubsection{Background}

Both SOS and SDSOS make use of the mapping $L: \mathbb{R}^{n} \rightarrow \mathbb{R}^{\left(\begin{array}{c}n+d \\ d\end{array}\right)}$ that takes a vector $\boldsymbol{x}$ to the vector containing all monomials of degree at-most- $d$ evaluated at $\boldsymbol{x}$. Specifically, these methods try to express a polynomial $p$ of degree $2 d$ as $p(\boldsymbol{x})=L(\boldsymbol{x})^{\top} \boldsymbol{M} L(\boldsymbol{x})$ for an appropriate matrix $\boldsymbol{M}$. In the case of SOS, the matrix $\boldsymbol{M}$ must be positive semidefinite, and so SOS decompositions can be found by semidefinite programming. For SDSOS, the matrix $\boldsymbol{M}$ must be not just positive semidefinite, but also satisfy the stronger property of scaled diagonal dominance ${ }^{5}$ [ [2] shows that polynomials representable in this way are precisely those given by sums-of-binomial-squares. From a computational perspective, SDSOS uses the fact that a scaled diagonally dominant matrix of order $m$ can be represented by $m(m-1) / 2$ rotated second order cones.

SONC takes a very different approach to polynomial nonnegativity. It begins by considering polynomials where the exponent vectors form a simplicial circuit. Fix such a polynomial $f=$ $\operatorname{Poly}\left([\boldsymbol{\alpha}, \boldsymbol{\beta}],[\boldsymbol{c}, b]^{\top}\right)$ where $\boldsymbol{\beta}$ is the nonextremal element of the Newton polytope, $\boldsymbol{\alpha}_{i}$ are even, and $\boldsymbol{c}>0$. Then by Theorem 3.8 of [20], there exists a quantity $\Theta_{f}$ that is independent of $b$, for which $f$ is nonnegative iff

$$
\text { either } \quad|b| \leq \Theta_{f} \text { and } \boldsymbol{\beta} \notin(2 \mathbb{N})^{n} \quad \text { or } \quad b \geq-\Theta_{f} \text { and } \boldsymbol{\beta} \in(2 \mathbb{N})^{n} \text {. }
$$

\footnotetext{
${ }^{4}$ The existence of such convex combinations follows from the argument in Lemma 8.

${ }^{5}$ A symmetric matrix $\boldsymbol{M}$ is scaled-diagonally-dominant if there exists diagonal $\boldsymbol{D} \succ \mathbf{0}$ so that $\boldsymbol{D} \boldsymbol{M} \boldsymbol{D}^{\top}$ is diagonally dominant. The positive semidefiniteness of these matrices follows from Gershgorin's Circle Theorem.
} 
The map $f \mapsto \Theta_{f}$ is concave in $\boldsymbol{c}$ (taking the form of a weighted geometric mean), and in particular the constraint $|b| \leq \Theta_{f}$ can be represented efficiently. Given a general polynomial $f=\operatorname{Poly}(\boldsymbol{\alpha}, \boldsymbol{c})$, the SONC relaxation enumerates the simplicial circuits that could be induced by $\boldsymbol{\alpha}$, and attempts to write $\boldsymbol{c}$ as a sum of vectors supported over these circuits (see Section 5.2 of [12]). The trouble with this approach is that the number of circuits needed in a SONC relaxation can be exponential in the number of terms appearing in the polynomial. Consequently, all current descriptions of SONC in the literature are either of exponential size in general or employ inexact approximations.

\subsubsection{Expressive power}

Every SDSOS polynomial is a SAGE polynomial. To see why, one need only note that binomial squares satisfy the hypothesis of Corollary 17. As a result, the equivalence of SDSOS polynomials and sums-of-binomial-squares ensures that every SDSOS polynomial is a SAGE polynomial. The reverse inclusion is not true, with a prominent example being the Motzkin form

$$
m(\boldsymbol{x})=x_{1}^{2} x_{2}^{4}+x_{1}^{4} x_{2}^{2}+x_{3}^{3}-3 x_{1}^{2} x_{2}^{2} x_{3}^{2}
$$

which is SAGE, but not even SOS (let alone SDSOS).

From the definition of SAGE polynomials it is more or less immediate that SAGE polynomials contain all SONC polynomials. This is because polynomials supported on simplicial circuits (the building blocks of SONC) are trivially single-orthant dominated with simplicial Newton polytope. What is more surprising is that SAGE polynomials and SONC polynomials are equivalent, at least in the abstract sense that $C_{\mathrm{SAGE}}^{\mathrm{POLY}}\left(\boldsymbol{M}_{n, d}\right)$ and $C_{\mathrm{SONC}}(n, d)$ share the same extreme rays.

This equivalence can provide an alternative way of understanding a few of our earlier results. For example, our Theorem 10 from Section 4 is the signomial version of Corollary 7.5 from Iliman and de Wolff's work [19]. As another example, our Corollary 17 is equivalent to Theorem 2.6 from Iliman and de Wolff's [20].

\subsubsection{Computational efficiency}

As was mentioned earlier in this section, existing proposals for checking polynomial nonnegativity via the SONC formulation $[38,12]$ are either rely on approximations of the SONC cone or are based on formulations that are exponential in size. Our results give an efficient relative entropy description of the SONC cone by demonstrating an equivalence with SAGE polynomials (see (26)).

The comparisons between SAGE and SOS/SDSOS are somewhat indirect, since the underlying mathematical programs do not use the same primitive cones. A structural distinction is that neither SOS nor SDSOS can approximatel $C_{\mathrm{NNP}}(\boldsymbol{\alpha})$ without possibly introducing additional exponent vectors. As an example consider the polynomial $f(x, y)=1-2 x^{2} y^{2}+x^{8} / 4+y^{8} / 4$; this polynomial is nonnegative, and admits both SAGE and SDSOS decompositions. The trouble is that while SAGE simply verifies that $f$ is an AGE polynomial with four terms, SDSOS will require additional terms $+x^{4} y^{4}$ and $-x^{4} y^{4}$. The situation only gets worse with SOS: the reduction techniques described in [24] are not helpful when the exponent vectors $\boldsymbol{\alpha}$ are even and $\mathcal{P}(\boldsymbol{\alpha})$ is simplicial.

We must also consider the sheer size of the nonnegativity cone associated with SOS and SDSOS: for a degree $2 d$ polynomial, both of these methods require a symmetric matrix variable of order $\left(\begin{array}{c}n+d \\ d\end{array}\right)$. By contrast Equation (26) lets us check if an $m$-term polynomial Poly $(\boldsymbol{\alpha}, \boldsymbol{c})$ is SAGE in a degree-independent way: we need only solve a relative entropy program of size $O\left(m^{2}+n m\right)$. Thus for sparse polynomials, there seems to be little competition between SAGE and SOS/SDSOS in terms of tractability. 


\subsection{Extending SAGE polynomials to a hierarchy}

We conclude our discussion of SAGE relaxations for sparse polynomial optimization by briefly outlining natural hierarchies of convex relative entropy programs that are "degree-independent" to obtain improved bounds. Our development is based on a hierarchy for signomials that is described in $[9]$.

We assume the following standard form for a polynomial optimization problem with objective $f$ and constraint functions $\left\{g_{i}\right\}_{i=1}^{k}$ :

$$
(f, g)^{\star}=\inf \left\{f(\boldsymbol{x}): \boldsymbol{x} \text { in } \mathbb{R}^{n} \text { satisfies } g_{i}(\boldsymbol{x}) \geq \mathbf{0} \text { for } i=1, \ldots, k\right\} .
$$

Here, all polynomials are over a common set of exponents $\boldsymbol{\alpha}$, with $\boldsymbol{\alpha}_{1}=\mathbf{0}$.

The typical approach method for coping with constrained polynomial optimization problems such as this begins by replacing $\left\{g_{i}\right\}_{i=1}^{k}$ with a larger set $\left\{h_{i}\right\}_{i=1}^{\ell}$ obtained by taking various products of the $g_{i}$; this idea has a longer lineage in nonlinear optimization in which redundant constraints are added to nonconvex problems to obtain improved bounds via the Lagrange dual. We then form a generalized Lagrangian $\mathcal{L}=f-\gamma-\sum_{i=1}^{\ell} \lambda_{i} h_{i}$, where the $\lambda_{i}$ are globally nonnegative polynomials belonging to some tractable convex set $P$. From here we would like to solve

$$
(f, g)_{P, h}=\sup _{\gamma \in \mathbb{R}}\left\{\gamma: \lambda_{i} \text { in } P, \mathcal{L}=f-\gamma-\sum_{i=1}^{\ell} \lambda_{i} h_{i} \text { nonnegative }\right\}
$$

which would certainly yield $(f, g)_{P, h} \leq(f, g)^{\star}$. However, for tractability reasons we instead check some kind of sufficient condition that $\mathcal{L}$ is nonnegative.

We now describe natural choices based on SAGE polynomials for the set $P$ and for the nonnegativity condition. Consider operators $\mathcal{A}$ and $\mathcal{C}$ taking values $\mathcal{A}(\operatorname{Poly}(\boldsymbol{\alpha}, \boldsymbol{c}))=\boldsymbol{\alpha}$ and $\mathcal{C}(\operatorname{Poly}(\boldsymbol{\alpha}, \boldsymbol{c}))=$ $\boldsymbol{c}$ respectively. We shall say that the SAGE polynomial hierarchy is indexed by two parameters: $p$ and $q$. The parameter $p$ controls the complexity of Lagrange multipliers; when $p=0$, the Lagrange multipliers are simply $\lambda_{i} \geq 0$. For general $p$, the Lagrange multipliers are SAGE polynomials over exponents $\mathcal{A}\left(\operatorname{Poly}(\boldsymbol{\alpha}, \mathbf{1})^{p}\right)$. The parameter $q$ controls the number of constraints in the nonconvex primal problem: $\left\{h_{i}\right\}_{i=1}^{k^{q}}$ are obtained by taking all $q$-fold products of the $g_{i}$. Once the Lagrangian $\mathcal{L}$ is formed, it will be a polynomial over exponents $\hat{\boldsymbol{\alpha}}=\mathcal{A}\left(\operatorname{Poly}(\boldsymbol{\alpha}, \mathbf{1})^{p+q}\right)$, and we constrain the Lagrangian to be nonnegative by writing $\mathcal{C}(\mathcal{L}) \in C_{\mathrm{SAGE}}^{\mathrm{POLY}}(\hat{\boldsymbol{\alpha}})$. Naturally, as $p$ and $q$ increase, we obtain improved bounds at the expense of an increase in computation.

One can interpret this family of relaxations as a "sparse polynomial specialization" of the constrained signomial optimization hierarchy described in [9]; as in that paper, one can also appeal to representation theorems from the real algebraic geometry literature $[26,29,34,37,44]$ to prove that the hierarchy outlined above can provide arbitrarily accurate lower bounds of sparse polynomial optimization problems in which the constraint set is compact.

Our broader message here - beyond results on convergence to the optimal value of specific hierarchies - is that the above construction qualitatively differs from other hierarchies in the literature because the optimization problems encountered at every level of our construction depend only on the nonnegative lattice generated by the original exponent vectors $\boldsymbol{\alpha}$. The theoretical underpinnings of this sparsity-preserving hierarchy trace back to the decomposition result given by Theorem 2. Thus, it is possible to obtain entire families of relative entropy relaxations that are sparsity-preserving, which reinforces our message about the utility of relative entropy optimization for sparse polynomial problems. 


\section{Towards Necessary and Sufficient Conditions for SAGE versus Nonnegativity}

We conclude this paper with a discussion on the extent to which our results tightly characterize the distinction between SAGE and nonnegativity for signomials. This section is split into three parts. In the first part, we describe a process for identifying cases where $C_{\mathrm{SAGE}}(\boldsymbol{\alpha}) \subsetneq C_{\mathrm{NNS}}(\boldsymbol{\alpha})$. This process is illustrated with several examples which suggest that our results from Section 4 are essentially tight. Section 6.2 presents a formal conjecture regarding the ways in which our results might be improved. We conclude with Section 6.3, where we provide a novel dual formulation for when $C_{\mathrm{SAGE}}(\boldsymbol{\alpha})=C_{\mathrm{NNS}}(\boldsymbol{\alpha})$.

\subsection{Constructing examples of non-equality}

Given a matrix of exponent vectors $\boldsymbol{\alpha}$, we are interested in finding a coefficient vector $\boldsymbol{c}$ so that $f=\operatorname{Sig}(\boldsymbol{\alpha}, \boldsymbol{c})$ satisfies $f_{\mathrm{SAGE}}<f^{\star}$. If such $\boldsymbol{c}$ exists, then it is evident that $C_{\mathrm{SAGE}}(\boldsymbol{\alpha}) \neq C_{\mathrm{NNS}}(\boldsymbol{\alpha})$.

The naïve approach to this process would be to carefully construct signomials where the infimum $f^{\star}$ is known by inspection, to compute $f_{\mathrm{SAGE}}$, and then to test if the measured value $\left|f_{\mathrm{SAGE}}-f^{\star}\right|$ is larger would be possible from rounding errors alone. A serious drawback of this approach is that it can be quite difficult to construct $\boldsymbol{\alpha}$ and $\boldsymbol{c}$ where $f^{\star}$ is apparent, and yet $\left\{\boldsymbol{\alpha}_{i}: c_{i} \neq 0\right\}$ satisfy the properties for the conjecture under test.

To address this challenge, we appeal to the idea alluded to in Section 2 that SAGE provides a means of computing an entire sequence of lower bounds $\left(f_{\mathrm{SAGE}}^{(p)}\right)_{p \in \mathbb{N}}$. For details on this "SAGE hierarchy," we refer the reader to [9]. For our purposes, suffice it to say that

$$
f_{\mathrm{SAGE}}^{(p)} \doteq \sup \left\{\gamma: \operatorname{Sig}(\boldsymbol{\alpha}, \mathbf{1})^{p}(f-\gamma) \text { is } \operatorname{SAGE}\right\}
$$

defines a non-decreasing sequence bounded above by $f^{\star}$. Thus, while we cannot readily check if $\left|f_{\mathrm{SAGE}}-f^{\star}\right| \gg 0$, we can compute a few values of $f_{\mathrm{SAGE}}^{(p)}$ for $p>0$, and check if $\left|f_{\mathrm{SAGE}}^{(0)}-f_{\mathrm{SAGE}}^{(p)}\right| \gg 0 .^{6}$

The remainder of this section goes through case studies in which we probe the sensitivity our earlier theorems' conclusions to their stated assumptions. All computation was performed with CVXPY $[10,1]$ as an interface to the conic solver ECOS $[11,39]$. Code used to generate the CVXPY problems can be found at github.com/rileyjmurray/sigpy. Numerical precision is reported to the farthest decimal point where the primal and dual methods for computing $f_{\mathrm{SAGE}}^{(p)}$ agree.

Example 1. We test here whether it is possible to relax the assumption of simplicial Newton polytope in Theorem 10. Since every Newton polytope in $\mathbb{R}$ is trivially simplicial, the simplest signomials available to us are over $\mathbb{R}^{2}$. With that in mind, consider

$$
\boldsymbol{\alpha}=\left[\begin{array}{llllll}
0 & 2 & 1 & 0 & 0 & 2 \\
0 & 0 & 0 & 2 & 1 & 2
\end{array}\right]
$$

This choice of $\boldsymbol{\alpha}$ is particularly nice, because were it not for the last column $\boldsymbol{\alpha}_{6}=[2,2]^{\top}$, we would very clearly have $C_{\mathrm{SAGE}}(\boldsymbol{\alpha})=C_{\mathrm{NNS}}(\boldsymbol{\alpha})$. As for the coefficient vector $\boldsymbol{c}$, we tested a few values before finding

$$
\boldsymbol{c}=[0,3,-4,2,-2,1]^{\top},
$$

\footnotetext{
${ }^{6}$ For completeness, we could also use heuristic optimization procedures (e.g. gradient descent with multiple random initializations) in the hopes of finding a point $\boldsymbol{x}$ with $f_{\mathrm{SAGE}}^{(p)}=f(\boldsymbol{x})$. Of course if we did find such a point, it would follow that $f_{\mathrm{SAGE}}^{(p)}=f^{\star}$.
} 
which resulted in $f_{\mathrm{SAGE}}^{(0)} \approx-1.83333$, and $f_{\mathrm{SAGE}}^{(1)} \approx-1.746505595=f^{\star}$. Because the absolute deviation $\left|f_{\mathrm{SAGE}}-f^{\star}\right| \approx 0.08682$ is much larger than the precision to which we solved these relaxations, we conclude that $C_{\mathrm{SAGE}}(\boldsymbol{\alpha}) \neq C_{\mathrm{NNS}}(\boldsymbol{\alpha})$ for this choice of $\boldsymbol{\alpha}$.

Example 2. Let us reinforce the conclusion from Example 1. Applying a 180 degree rotation about the point $(1,1)$ to the columns of $\boldsymbol{\alpha}$, we obtain

$$
\boldsymbol{\alpha}=\left[\begin{array}{llllll}
0 & 2 & 0 & 2 & 1 & 2 \\
0 & 0 & 2 & 2 & 2 & 1
\end{array}\right]
$$

We then choose the coefficient vector in a manner informed by the theory developed in Section 6.3

$$
\boldsymbol{c}=[0,1,1,1.9,-2,-2]^{\top}
$$

which subsequently defines $f=\operatorname{Sig}(\boldsymbol{\alpha}, \boldsymbol{c})$. In this case, the primal formulation for $f_{\mathrm{SAGE}}$ is demonstrably infeasible, and the dual formulation has an improving direction (i.e. is unbounded), hence we have $f_{\mathrm{SAGE}}^{(0)}=-\infty$. Meanwhile, the second level of the SAGE hierarchy produces $f_{\mathrm{SAGE}}^{(1)} \approx-0.122211863=f^{\star}$. Thus in a very literal sense, the gap $\left|f_{\mathrm{SAGE}}-f^{\star}\right|$ could not be larger. ${ }^{7}$

Example 3. We know from Theorem 12 that any signomial with at most four terms is nonnegative if and only if it is SAGE. It is natural to wonder if in some very restricted setting (e.g. univariate signomials) the SAGE and nonnegativity cones would coincide for signomials with five or more terms. Perhaps the simplest example here is $\boldsymbol{\alpha}=[0,1,2,3,4]$. After some experimentation, the authors found that $f=\operatorname{Sig}(\boldsymbol{\alpha}, \boldsymbol{c})$ with $\boldsymbol{c}=[1,-4,7,-4,1]$ has $f_{\mathrm{SAGE}}^{(0)} \approx-0.3333333$ and $f_{\mathrm{SAGE}}^{(1)} \approx 0.2857720944$.

By appropriately appealing to the invariance properties of the SAGE and nonnegativity cones, a single counter-example generates a whole class of matrices $\boldsymbol{\alpha}$ for which $C_{\mathrm{SAGE}}(\boldsymbol{\alpha}) \neq C_{\mathrm{NNS}}(\boldsymbol{\alpha})$. In the case of Example 3, we can actually conclude that $C_{\mathrm{SAGE}}(\boldsymbol{\alpha})$ is a strict subset of $C_{\mathrm{NNS}}(\boldsymbol{\alpha})$ for every $1 \times 5$ matrix $\boldsymbol{\alpha}$ with five equispaced values.

These examples demonstrate that there are senses in which Theorems 10 through 12 cannot be meaningfully improved upon.

\subsection{A conjecture, under mild regularity conditions}

Despite the conclusion in the previous subsection, there are settings when we can prove $C_{\mathrm{SAGE}}(\boldsymbol{\alpha})=$ $C_{\mathrm{NNS}}(\boldsymbol{\alpha})$ in spite of $\boldsymbol{\alpha}$ not satisfying the assumptions of Theorem 12. For example, one case in which SAGE equals nonnegativity is when $\boldsymbol{\alpha}=[\mathbf{0}, \boldsymbol{I}, \boldsymbol{D}]$ where $\boldsymbol{D}$ is a diagonal matrix with diagonal entries in $(0,1)$. Here one proves equality as follows: for each possible sign pattern of $\boldsymbol{c} \in C_{\mathrm{NNS}}(\boldsymbol{\alpha})$, there exists a lower dimensional simplicial face $F$ of $\mathcal{P}(\boldsymbol{\alpha})$ upon which we invoke Theorem 10, and for which the remaining exponents (those outside of $F$ ) have positive coefficients. We know that the signomial induced by the exponents outside of $F$ is trivially SAGE, and so by Theorem 11 we conclude $\boldsymbol{c} \in C_{\mathrm{SAGE}}(\boldsymbol{\alpha})$. As this holds for all possible sign patterns on $\boldsymbol{c}$ in $C_{\mathrm{NNS}}(\boldsymbol{\alpha})$, we have $C_{\mathrm{SAGE}}(\boldsymbol{\alpha})=C_{\mathrm{NNS}}(\boldsymbol{\alpha})$. However, this case is somewhat degenerate, and we wish to exclude it in our discussion via some form of regularity on $\boldsymbol{\alpha}$.

The most natural regularity condition on $\boldsymbol{\alpha}$ would be that it admits only the trivial partition, and indeed we focus on the case when every $\boldsymbol{\alpha}_{i}$ belongs to either ext $\mathcal{P}(\boldsymbol{\alpha})$ or int $\mathcal{P}(\boldsymbol{\alpha})$. In this setting we have the following corollary of Theorem 12 .

\footnotetext{
${ }^{7}$ To our knowledge this is the first recorded case where $f_{\mathrm{SAGE}}=-\infty$, but the second level of the hierarchy is exact.
} 
Corollary 21. If $\mathcal{P}(\boldsymbol{\alpha})$ is full dimensional with either

1. at most one interior exponent, or

2. $n+1$ extreme points and at most two interior exponents

then $C_{\mathrm{SAGE}}(\boldsymbol{\alpha})=C_{\mathrm{NNS}}(\boldsymbol{\alpha})$.

Along with this corollary, we present a conjecture for the reader's consideration.

Conjecture 22. If $\mathcal{P}(\boldsymbol{\alpha})$ has every $\boldsymbol{\alpha}_{i}$ in either ext $\mathcal{P}(\boldsymbol{\alpha})$ or int $\mathcal{P}(\boldsymbol{\alpha})$, but $\boldsymbol{\alpha}$ does not satisfy the hypothesis of Corollary 21, then $C_{\mathrm{SAGE}}(\boldsymbol{\alpha}) \neq C_{\mathrm{NNS}}(\boldsymbol{\alpha})$.

Note that when $\boldsymbol{\alpha}$ satisfies the stated assumptions and and further has some $\boldsymbol{\alpha}_{i}=\mathbf{0}$ in the interior, Theorem 5 ensures that $f=\operatorname{Sig}(\boldsymbol{\alpha}, \boldsymbol{c})$ can have $f_{\mathrm{SAGE}}$ deviate from $f^{\star}$ only by a finite amount. To overcome a potential obstacle posed by this result in the resolution of the above conjecture, one can also consider modifying the hypotheses of the conjecture to require that all $\boldsymbol{\alpha}_{i}$ lie in the relative interior of the Newton polytope.

To finish discussion on Conjecture 22, we provide empirical support with the following examples.

Example 4. Let $f$ be a signomial in two variables with

$$
\left[\begin{array}{c}
\boldsymbol{\alpha} \\
\hline \boldsymbol{c}^{\top}
\end{array}\right]=\left[\begin{array}{cccccc}
0 & 1 & 0 & 0.30 & 0.21 & 0.16 \\
0 & 0 & 1 & 0.58 & 0.08 & 0.54 \\
\hline 33.94 & 67.29 & 1 & 38.28 & -57.75 & -40.37
\end{array}\right]
$$

Then $f_{\mathrm{SAGE}}=-24.054866<f_{\mathrm{SAGE}}^{(1)}=-21.31651$. This example provides the minimum number of interior exponents needed to be relevant to Conjecture 22 in the simplicial case.

Example 5. Let $f$ be a signomial in two variables with

$$
\left[\begin{array}{c}
\boldsymbol{\alpha} \\
\hline \boldsymbol{c}^{\top}
\end{array}\right]=\left[\begin{array}{cccccc}
0 & 1 & 0 & 2 & 0.52 & 1.30 \\
0 & 0 & 1 & 2 & 0.15 & 1.38 \\
\hline 0.31 & 0.85 & 2.55 & 0.65 & -1.48 & -1.73
\end{array}\right] .
$$

then $f_{\mathrm{SAGE}}=0.00354263<f_{\mathrm{SAGE}}^{(1)}=0.13793126$. This signomial has the minimum number of interior exponents needed to be relevant to Conjecture 22 in the nonsimplicial case.

\subsection{A dual characterization of SAGE versus nonnegativity}

In this section, we provide a general necessary and sufficient dual characterization in terms of certain moment-type mappings for the question of $C_{\mathrm{SAGE}}(\boldsymbol{\alpha})=C_{\mathrm{NNS}}(\boldsymbol{\alpha})$. We begin with the following proposition (proven in the appendix).

Proposition 23. If $\boldsymbol{\alpha}$ has $\boldsymbol{\alpha}_{1}=\mathbf{0}$, then the following are equivalent:

1. For every vector $\boldsymbol{c}$, the function $f=\operatorname{Sig}(\boldsymbol{\alpha}, \boldsymbol{c})$ satisfies $f^{\star}=f_{\mathrm{SAGE}}$.

2. $C_{\mathrm{NNS}}(\boldsymbol{\alpha})=C_{\mathrm{SAGE}}(\boldsymbol{\alpha})$.

3. $\left\{\boldsymbol{v}: v_{1}=1, \boldsymbol{v}\right.$ in $\left.C_{\mathrm{SAGE}}(\boldsymbol{\alpha})^{\dagger}\right\} \subset \operatorname{cl} \operatorname{conv} \exp \mathcal{R}\left(\boldsymbol{\alpha}^{\top}\right)$. 
Our dual characterization consists of two new sets, both parameterized by $\boldsymbol{\alpha}$. The first of these sets relates naturally to the third condition in Proposition 23. Formally, the moment preimage of some exponent vectors $\boldsymbol{\alpha}$ is the set

$$
T(\boldsymbol{\alpha}) \doteq \log \operatorname{clconv} \exp \mathcal{R}\left(\boldsymbol{\alpha}^{\boldsymbol{\top}}\right)
$$

Here, we extend the logarithm to include $\log 0=-\infty$ in the natural way. The second set appearing in our dual characterization is defined less explicitly. For a given $\boldsymbol{\alpha}$, we say that $S(\boldsymbol{\alpha})$ is a set of $S A G E$-feasible slacks if $f=\operatorname{Sig}(\boldsymbol{\alpha}, \boldsymbol{c})$ has

$$
f_{\mathrm{SAGE}}=\inf \left\{\boldsymbol{c}^{\boldsymbol{\top}} \exp \boldsymbol{y}: \boldsymbol{y} \text { in } \mathcal{R}\left(\boldsymbol{\alpha}^{\boldsymbol{\top}}\right)+S(\boldsymbol{\alpha})\right\}
$$

for every $\boldsymbol{c}$ in $\mathbb{R}^{m}$.

Theorem 24. Let $\boldsymbol{\alpha}$ have $\boldsymbol{\alpha}_{1}=\mathbf{0}$, and let $S(\boldsymbol{\alpha})$ be any set of $S A G E$-feasible slacks over exponents $\boldsymbol{\alpha}$. Then $C_{\mathrm{SAGE}}(\boldsymbol{\alpha})=C_{\mathrm{NNS}}(\boldsymbol{\alpha})$ iff $S(\boldsymbol{\alpha}) \subset T(\boldsymbol{\alpha})$.

Theorem 24. To keep notation compact write $U=\mathcal{R}\left(\boldsymbol{\alpha}^{\top}\right)$ and $S=S(\boldsymbol{\alpha})$. Also, introduce $W=$ $\left\{\boldsymbol{v}: v_{1}=1, \boldsymbol{v}\right.$ in $\left.C_{\mathrm{SAGE}}(\boldsymbol{\alpha})^{\dagger}\right\}$ to describe the feasible set to the dual formulation for $f_{\mathrm{SAGE}}$. By the supporting-hyperplane characterizations of convex sets, the definitions of $S$ and $W$ ensure the relation

$$
W=\operatorname{clconv} \exp (\mathcal{U}+S) .
$$

Thus by the equivalence of 1 and 3 in Proposition 23, it follows that all SAGE relaxations will be exact if and only if $\exp (U+S) \subset \operatorname{cl} \operatorname{conv} \exp U$. We apply a pointwise logarithm to write the latter condition as $U+S \subset \log \mathrm{cl} \operatorname{conv} \exp U$.

Now we prove that $T \doteq \log \operatorname{clconv} \exp U$ is invariant under translation by vectors in $U$. It suffices to show that $\exp (\boldsymbol{v}+T)=\exp T$ for all vectors $\boldsymbol{v}$ in $U$. Fixing $\boldsymbol{v}$ in $U$ we have

$$
\begin{aligned}
\exp (\boldsymbol{v}+T) & =\exp (\boldsymbol{v}) \odot \exp (T) \\
& =\exp (\boldsymbol{v}) \odot \operatorname{cl} \operatorname{conv} \exp (U) \\
& =\operatorname{cl} \operatorname{conv} \exp (\boldsymbol{v}+U) \\
& =\operatorname{cl} \operatorname{conv} \exp (U)=\exp (T)
\end{aligned}
$$

as claimed. This translation invariance establishes that $U+S \subset \log$ cl conv $\exp U$ is equivalent to $S \subset \log \operatorname{clconv} \exp U$, and in turn that condition 1 of Proposition 23 holds if and only if $S \subset \log$ cl conv $\exp U$. The claim now follows by the equivalence of 1 and 2 in Proposition 23 .

It is the authors' hope that Theorem 24 may help future efforts to resolve Conjecture 22. A starting point in understanding the moment preimage could be to use cumulant generating functions from probability theory. For constructing sets of SAGE-feasible slacks, one might use a change-ofvariables argument similar to that seen in the proof of Theorem 10.

\section{Acknowledgments}

V.C. would like to acknowledge helpful conversations with Parikshit Shah, particularly on the connections between SAGE and SDSOS polynomials. R.M. was supported in part by NSF grant CCF-1637598 and by an NSF Graduate Research Fellowship. V.C. was supported in part by NSF grants CCF-1350590 and CCF-1637598, AFOSR grant FA9550-16-1-0210, and a Sloan Research Fellowship. A.W. was supported in part by NSF grant CCF-1637598. 


\section{References}

[1] Akshay Agrawal, Robin Verschueren, Steven Diamond, and Stephen Boyd. A rewriting system for convex optimization problems. Journal of Control and Decision, 5(1):42-60, 2018.

[2] Amir Ali Ahmadi and Anirudha Majumdar. Dsos and sdsos optimization: Lp and socp-based alternatives to sum of squares optimization. In 2014 48th Annual Conference on Information Sciences and Systems (CISS), pages 1-5, March 2014.

[3] Amir Ali Ahmadi and Anirudha Majumdar. Dsos and sdsos optimization: More tractable alternatives to sum of squares and semidefinite optimization, 2017.

[4] MOSEK ApS. MOSEK 9.0.0.35(ALPHA), 2018.

[5] Tomáš Bajbar and Oliver Stein. Coercive polynomials and their newton polytopes. SIAM Journal on Optimization, 25(3):1542-1570, jan 2015.

[6] D. N. Bernshtein. The number of roots of a system of equations. Functional Analysis and Its Applications, 9(3):183-185, 1979.

[7] Stephen Boyd, Seung-Jean Kim, Lieven Vandenberghe, and Arash Hassibi. A tutorial on geometric programming. Optimization and Engineering, 8(1):67-127, apr 2007.

[8] V. Cerone, D. Piga, and D. Regruto. Bounded error identification of hammerstein systems through sparse polynomial optimization. Automatica, 48(10):2693 - 2698, 2012.

[9] Venkat Chandrasekaran and Parikshit Shah. Relative entropy relaxations for signomial optimization. SIAM Journal on Optimization, 26(2):11471173, 2016.

[10] Steven Diamond and Stephen Boyd. CVXPY: A Python-embedded modeling language for convex optimization. Journal of Machine Learning Research, 17(83):1-5, 2016.

[11] A. Domahidi, E. Chu, and S. Boyd. ECOS: An SOCP solver for embedded systems. In European Control Conference (ECC), pages 3071-3076, 2013.

[12] Mareike Dressler, Sadik Iliman, and Timo De Wolff. A positivstellensatz for sums of nonnegative circuit polynomials. SIAM Journal on Applied Algebra and Geometry, 1(1):536555, 2017.

[13] Chao Feng, C M Lagoa, and M Sznaier. Hybrid system identification via sparse polynomial optimization. In Proceedings of the 2010 American Control Conference. IEEE, jun 2010.

[14] Karin Gatermann and Matthias Wolfrum. Bernstein's second theorem and viro's method for sparse polynomial systems in chemistry. Advances in Applied Mathematics, 34(2):252-294, feb 2005.

[15] B. Ghaddar, J. Marecek, and M. Mevissen. Optimal power flow as a polynomial optimization problem. IEEE Transactions on Power Systems, 31(1):539-546, Jan 2016.

[16] M. Ghasemi, J. B. Lasserre, and M. Marshall. Lower bounds on the global minimum of a polynomial. Computational Optimization and Applications, 57(2):387-402, sep 2013.

[17] Mehdi Ghasemi and Murray Marshall. Lower bounds for polynomials using geometric programming. SIAM Journal on Optimization, 22(2):460-473, jan 2012. 
[18] Wenrui Hao, Jonathan D. Hauenstein, Bei Hu, Timothy McCoy, and Andrew J. Sommese. Computing steady-state solutions for a free boundary problem modeling tumor growth by stokes equation. Journal of Computational and Applied Mathematics, 237(1):326 - 334, 2013.

[19] Sadik Iliman and Timo De Wolff. Amoebas, nonnegative polynomials and sums of squares supported on circuits. Research in the Mathematical Sciences, 3(1):9, Mar 2016.

[20] Sadik Iliman and Timo De Wolff. Lower bounds for polynomials with simplex newton polytopes based on geometric programming. SIAM Journal on Optimization, 26(2):11281146, 2016.

[21] Orcun Karaca, Georgios Darivianakis, Paul Beuchat, Angelos Georghiou, and John Lygeros. The repop toolbox: Tackling polynomial optimization using relative entropy relaxations. IFACPapersOnLine, 50(1):11652 - 11657, 2017. 20th IFAC World Congress.

[22] A.G. Khovanskii. Fewnomials and pfaff manifolds. In Proceedings of the International Congress of Mathematicians, volume 1, page 2, 1983.

[23] A.G. Khovanskii. Fewnomials, volume 88. American Mathematical Soc., 1991.

[24] Masakazu Kojima, Sunyoung Kim, and Hayato Waki. Sparsity in sums of squares of polynomials. Mathematical Programming, 103(1):45-62, dec 2004.

[25] Anatoli G. Kouchnirenko. Polydres de newton et nombres de milnor. Inventiones mathematicae, 32:1-32, 1976.

[26] Jean-Louis Krivine. Anneaux préordonnés. Journal d'analyse mathématique, 12:p. 307-326, 1964.

[27] Jean B. Lasserre. Global optimization with polynomials and the problem of moments. SIAM Journal on Optimization, 11(3):796-817, jan 2001.

[28] Costas D. Maranas and Christodoulos A. Floudas. Global optimization in generalized geometric programming. Computers $\&$ Chemical Engineering, 21(4):351-369, dec 1997.

[29] Murray Marshall. Positive Polynomials and Sums of Squares. American Mathematical Society, feb 2008.

[30] Jiawang Nie and James Demmel. Sparse SOS relaxations for minimizing functions that are summations of small polynomials. SIAM Journal on Optimization, 19(4):1534-1558, jan 2009.

[31] James Oxley. What is a matroid? https://www.math.lsu.edu/ oxley/survey4.pdf, 2014.

[32] N. Ozay, M. Sznaier, and C. Lagoa. Model (in) validation of switched arx systems with unknown switches and its application to activity monitoring. In 49th IEEE Conference on Decision and Control (CDC), pages 7624-7630, Dec 2010.

[33] Pablo Parillo. Structured semidefinite programs and semialgebraic geometry methods in robustness and optimization. $\mathrm{PhD}$ thesis, California Institute of Technology, Pasadena, CA, 5 2000 .

[34] Mihai Putinar. Positive polynomials on compact semi-algebraic sets. Indiana University Mathematics Journal, 42(3):969-984, 1993. 
[35] Bruce Reznick. Extremal PSD forms with few terms. Duke Mathematical Journal, 45(2):363374, jun 1978.

[36] Bruce Reznick. Forms derived from the arithmetic-geometric inequality. Mathematische Annalen, 283(3):431-464, 1989.

[37] Konrad Schmdgen. The k-moment problem for compact semi-algebraic sets. Mathematische Annalen, 289(2):203-206, 1991.

[38] Henning Seidler and Timo De Wolff. An experimental comparison of sonc and sos certificates for unconstrained optimization, 2018.

[39] Santiago Akle Serrano. Algorithms for unsymmetric cone optimization and an implementation for problems with the exponential cone. PhD thesis, Stanford University, Palo Alto, CA, 2015.

[40] Peiping Shen. Linearization method of global optimization for generalized geometric programming. Applied Mathematics and Computation, 162(1):353-370, mar 2005.

[41] Hanif D. Sherali. Global optimization of nonconvex polynomial programming problems having rational exponents. Journal of Global Optimization, 12(3):267-283, 1998.

[42] N. Z. Shor. Class of global minimum bounds of polynomial functions. Cybernetics, 23(6):731$734,1988$.

[43] Anders Skajaa and Yinyu Ye. A homogeneous interior-point algorithm for nonsymmetric convex conic optimization. Mathematical Programming, 150(2):391-422, may 2014.

[44] Gilbert Stengle. A nullstellensatz and a positivstellensatz in semialgebraic geometry. Mathematische Annalen, 207(2):87-97, jun 1974.

[45] Hayato Waki, Sunyoung Kim, Masakazu Kojima, and Masakazu Muramatsu. Sums of squares and semidefinite program relaxations for polynomial optimization problems with structured sparsity. SIAM Journal on Optimization, 17(1):218-242, jan 2006.

[46] Y. Wang, M. Orshansky, and C. Caramanis. Enabling efficient analog synthesis by coupling sparse regression and polynomial optimization. In 2014 51st ACM/EDAC/IEEE Design Automation Conference (DAC), pages 1-6, June 2014. 


\section{Appendix}

\subsection{Numerical Implementation}

An important contribution of this work is sigpy: a Python-based implementation of the SAGE hierarchy that is readily testable, highly extensible, and human-readable. Virtually all of the sigpy package consists of two extensively commented modules containing less than 700 lines of code in total.

The signomials.py module defines a Signomial class; Signomial objects provide a symbolic representation for $f=\operatorname{Sig}(\boldsymbol{\alpha}, \boldsymbol{c})$. Signomial objects are operator-overloaded (for addition, subtraction, multiplication, and exponentiation), and allow coefficients $c_{i}$ as any datatype implementing addition, subtraction, and multiplication. This module depends only on numpy.

The sage.py module adds SAGE support to CVXPY. It allows the user to effortlessly construct explicit mathematical programs for SAGE relaxations (both constrained and unconstrained) in primal and dual forms. By integrating with CVXPY, sage.py provides easy access to exponential cone solvers such as ECOS, SCS, and MOSEK 9.

Let us work through an example; suppose you want to compute $f_{\mathrm{SAGE}}$ for

$f=\operatorname{sigpy} \cdot$ Signomial (alpha, c) .

This can be done directly with provided helper functions

prob = sigpy.sage_primal $(f$, level $=0)$

f_sage $=$ prob.solve(),

but just as well, you can construct the problem from scratch

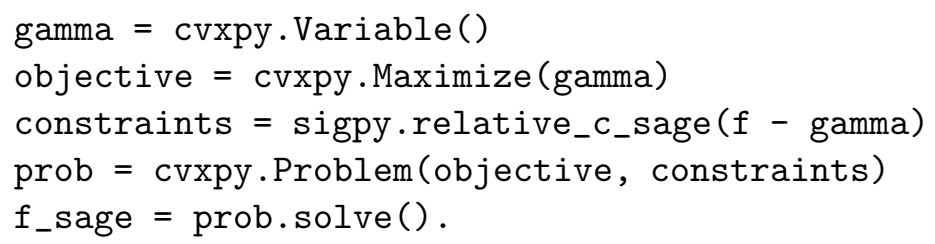

The function name relative_c_sage is chosen because it returns constraints for the reduced SAGE cone, as implied by Theorem 2 .

The implementation of primal and dual forms of SAGE relaxations is important, because exponential cone solvers have yet to fully mature. In the unittests written for sigpy, we always separately solve the primal and dual problems

f_sage_prim = sigpy.sage_primal (f, level=p)

f_sage_dual = sigpy.sage_dual $(f, l e v e l=p)$

and check that $f_{-}$sage_prim is sufficiently close to $f_{-}$sage_dual. Since we have a proof that strong duality always holds for these problems, any nontrivial difference between the numeric primal and dual values can be traced back to infeasible or suboptimal solutions. The user can be wise to the possibility of such numeric issues by calling prob.solve (verbose=True) on the CVXPY problems. The output of interior point solvers ECOS and MOSEK will reliably highlight inaccurate nearoptimal solutions, while SCS will display error metrics that the user should asses.

The source code for sigpy and additional documentation (particularly for SAGE polynomial relaxations) can be found at github.com/rileyjmurray/sigpy. 


\subsection{Proof of Proposition 1}

In Section 2 it was asserted that strong duality held between optimization problems (5) and (6). Here we prove a more general result with two lemmas. In what follows, "co" is an operator that computes a set's conic hull.

Lemma 25. Fix a closed convex cone $K$ in $\mathbb{R}^{n}$. If $\boldsymbol{a}$ in $K^{\dagger}$ is such that

$$
X \doteq\left\{\boldsymbol{x}: \boldsymbol{a}^{\top} \boldsymbol{x}=1, \boldsymbol{x} \text { in } K\right\}
$$

is nonempty, then $\operatorname{cl} \operatorname{co} X=K$.

Proof. Certainly the conic hull of $X$ is contained within $K$, and the same is true of its closure. The task is to show that every $\boldsymbol{x}$ in $K$ also belongs to cl co $X$; we do this by case analysis on $b \doteq \boldsymbol{a}^{\top} \boldsymbol{x}$.

By the assumptions $\boldsymbol{a} \in K^{\dagger}$ and $\boldsymbol{x} \in K$, we must have $b \geq 0$. If $b$ is positive then the scaling $\tilde{\boldsymbol{x}} \doteq \boldsymbol{x} / b$ belongs to $K$ and satisfies $\boldsymbol{a}^{\top} \tilde{\boldsymbol{x}}=1$. That is, $b>0$ gives us $\tilde{\boldsymbol{x}}$ in $X$. Simply undo this scaling to recover $\boldsymbol{x}$ and conclude $\boldsymbol{x} \in \operatorname{co} X$. Now suppose $b=0$ Here we consider the sequence of points $\boldsymbol{y}_{n} \doteq \boldsymbol{x}_{0}+n \boldsymbol{x}$, where $\boldsymbol{x}_{0}$ is a fixed but otherwise arbitrary element of $X$. Each point $\boldsymbol{y}_{n}$ belongs to $K$, and has $\boldsymbol{a}^{\top} \boldsymbol{y}_{n}=1$, hence the $\boldsymbol{y}_{n}$ are contained in $X$. It follows that the scaled points $\boldsymbol{y}_{n} / n$ are contained in cl co $X$, and the same must be true of their $\operatorname{limit}_{\lim } \rightarrow \infty \boldsymbol{y}_{n} / n=\boldsymbol{x}$.

Since $\boldsymbol{x}$ in $K$ was arbitrary, we have $\operatorname{cl} \operatorname{co} X=K$.

Lemma 26. Let $C$ be a closed convex cone, and let $\boldsymbol{a}$ be a nonzero vector in $C^{\dagger}$. Then the primal dual pair

$$
\begin{aligned}
& f_{\mathrm{p}}=\sup \{\gamma: \boldsymbol{c}-\gamma \boldsymbol{a} \text { in } C\} \text { and } \\
& f_{\mathrm{d}}=\inf \left\{\boldsymbol{c}^{\top} \boldsymbol{v}: \boldsymbol{a}^{\top} \boldsymbol{v}=1, \boldsymbol{v} \text { in } C^{\dagger}\right\}
\end{aligned}
$$

exhibits strong duality.

Proof. By assumption that $\boldsymbol{a}$ is a nonzero vector in $C^{\dagger}$, the dual feasible set $\left\{\boldsymbol{v}: \boldsymbol{a}^{\top} \boldsymbol{v}=1, \boldsymbol{v}\right.$ in $\left.C^{\dagger}\right\}$ is nonempty. Since the dual problem is feasible, a proof that $f_{\mathrm{d}}=f_{\mathrm{p}}$ can be divided into the cases $f_{\mathrm{d}}=-\infty$, and $f_{\mathrm{d}}$ in $\mathbb{R}$. The proof in former case is trivial; weak duality combined with $f_{\mathrm{p}} \geq-\infty$ gives $f_{\mathrm{d}}=f_{\mathrm{p}}$. In the latter case we prove $f_{\mathbf{p}} \geq f_{\mathbf{d}}$ by showing that $\boldsymbol{c}^{\star} \doteq \boldsymbol{c}-f_{\mathrm{d}} \boldsymbol{a}$ belongs to $C$.

To prove $\boldsymbol{c}^{\star} \in C$ we will appeal to Lemma 25 with $K \doteq C^{\dagger}$. Clearly the set $X=\left\{\boldsymbol{v}: \boldsymbol{a}^{\top} \boldsymbol{v}=\right.$ $1, \boldsymbol{v}$ in $K\}$ is precisely the [nonempty] feasible set for computing $f_{\mathrm{d}}$, and so from the definition of $f_{\mathrm{d}}$ we have $\boldsymbol{c}^{\star \top} \boldsymbol{v} \geq 0$ for all $\boldsymbol{v}$ in $X$. The inequality also applies to any $\boldsymbol{v}$ in cl co $X$, which by Lemma 25 is equal to $K^{\dagger}$. Therefore the definition of $f_{\mathrm{d}}$ ensures $\boldsymbol{c}^{\star}$ is in $K^{\dagger}$. Using $K^{\dagger} \equiv C$, we have the desired result.

Strong duality in computation of $f_{\mathrm{SAGE}}$ for $f=\operatorname{Sig}(\boldsymbol{\alpha}, \boldsymbol{c})$ readily follows from Lemma 26. Letting $\mathcal{N}=\left\{i: c_{i}<0\right\}$, simply take $C=C_{\mathrm{SAGE}}(\boldsymbol{\alpha})$ or $C=\sum_{i \in \mathcal{N} \cup\{1\}} C_{\mathrm{AGE}}(\boldsymbol{\alpha}, i, \mathcal{N})$, and use $\boldsymbol{a}=\boldsymbol{e}_{1}$. What's more, with appropriate bookkeeping one can use Lemma 26 to prove strong duality in computation of $f_{\mathrm{SAGE}}^{(p)}$ for any nonnegative integer $p$ !

\subsection{Proof of Proposition 23}

Proof. The cases $(2) \Rightarrow(1)$ and $(3) \Rightarrow(1)$ are easy.

$\neg(2) \Rightarrow \neg(1)$. Because $C_{\mathrm{NNS}}(\boldsymbol{\alpha})$ and $C_{\mathrm{SAGE}}(\boldsymbol{\alpha})$ are full dimensional closed convex sets, the condition $C_{\mathrm{SAGE}}(\boldsymbol{\alpha}) \neq C_{\mathrm{NNS}}(\boldsymbol{\alpha})$ implies that $C_{\mathrm{NNS}}(\boldsymbol{\alpha}) \backslash C_{\mathrm{SAGE}}(\boldsymbol{\alpha})$ has nonempty interior. Assuming 
this condition, fix a vector $\tilde{\boldsymbol{c}}$ and a radius $r$ such that $B(\tilde{\boldsymbol{c}}, r) \subset C_{\mathrm{NNS}}(\boldsymbol{\alpha}) \backslash C_{\mathrm{SAGE}}(\boldsymbol{\alpha}){ }^{8}$ This allows us to strictly separate $\tilde{\boldsymbol{c}}$ from $C_{\mathrm{SAGE}}(\boldsymbol{\alpha})$, which establishes $f^{\star} \geq f_{\mathrm{SAGE}}+r>f_{\mathrm{SAGE}}$.

$(1) \Rightarrow(3)$. Now suppose that $f^{\star}=f_{\mathrm{SAGE}}$ for all relevant $f$. In this case, the function $\boldsymbol{c} \mapsto$ $\inf \left\{\boldsymbol{c}^{\top} \boldsymbol{x}: \boldsymbol{x} \in \Omega\right\}$ is the same for $\Omega=\mathrm{cl}$ conv $\exp \mathcal{R}\left(\boldsymbol{\alpha}^{\top}\right)$ or $\Omega=\left\{\boldsymbol{v}: v_{1}=1\right.$ and $\boldsymbol{v}$ in $\left.C_{\mathrm{SAGE}}(\boldsymbol{\alpha})^{\star}\right\}$. This function completely determines the set of all half spaces containing $\Omega$. Since $\Omega$ is closed and convex, it is precisely equal to the intersection of all half spaces containing it; the result follows.

\footnotetext{
${ }^{8} B(\boldsymbol{x}, d)$ is $\ell_{2}$ ball centered at $\boldsymbol{x}$ of radius $d$.
} 